\title{
Weighted Stepanov-Like Pseudoperiodicity and Applications
}

\author{
Zhinan Xia \\ Department of Applied Mathematics, Zhejiang University of Technology, Hangzhou, Zhejiang 310023, China \\ Correspondence should be addressed to Zhinan Xia; xiazn299@zjut.edu.cn
}

Received 28 June 2013; Revised 28 October 2013; Accepted 28 October 2013; Published 20 January 2014

Academic Editor: Carlos Lizama

Copyright (C) 2014 Zhinan Xia. This is an open access article distributed under the Creative Commons Attribution License, which permits unrestricted use, distribution, and reproduction in any medium, provided the original work is properly cited.

By the weighted ergodic space, we propose a new class of functions called weighted Stepanov-like pseudoperiodic function and explore its properties. Furthermore, the existence and uniqueness of the weighted pseudoperiodic solution to fractional integrodifferential equations and nonautonomous differential equations are investigated. Some interesting examples are presented to illustrate the main findings.

\section{Introduction}

The study of the existence of periodic solutions is one of the most interesting and important topics in the qualitative theory of differential equation. Many authors have made important contributions to this theory. Recently, in [1], the concept of weighted pseudoperiodicity, which generalizes the notion of periodicity and pseudoperiodicity, is introduced and studied. On the other hand, Stepanov $[2,3]$ introduced a class of generalized almost periodic functions, for which continuity fails, and only measurability and integrability in the sense of Lebesgue are required. Since then, almost automorphy, pseudo-almost-periodicity, pseudo-almost-automorphy, and so forth are generalized in the Stepanov sense one can see [46] for more details on this topics.

Motivated by the above mentioned papers, in this paper, we introduced a new class of functions called weighted Stepanov-like pseudoperiodic function, which generalizes the notation of weighted pseudoperiodic function. We systematically explore the properties of the weighted Stepanovlike pseudoperiodic function in general Banach space including a composition result.

The rapid development of the theory of integrodifferential equations has been strongly promoted by the large number of applications in physics, engineering, biology, and other subjects. This type of equations has received much attention in recent years and the general asymptotic behavior of solutions is at present an active of research [7-12]. To the best of our knowledge, there is no work reported in literature on weighted pseudoperiodicity for fractional integrodifferential equations in $1<\alpha<2$. Furthermore, the existence and uniqueness of weighted pseudoperiodic solutions for nonautonomous differential equations are quite new and untreated topic. This is one of the key motivations of this study.

The paper is organized as follows. In Section 2, some notations and preliminary results are presented. Next, we propose a new class of functions called weighted Stepanov-like pseudoperiodic function, explore its properties, and establish the composition theorem. Sections 3 and 4 are devoted to the existence and uniqueness of weighted pseudoperiodic solutions to a class of fractional integrodifferential equations and nonautonomous differential equations, respectively. In Section 5, we provide some examples to illustrate our main results.

\section{Preliminaries and Basic Results}

Let $(X,\|\cdot\|),(Y,\|\cdot\|)$ be two Banach spaces and $\mathbb{N}, \mathbb{Z}$, $\mathbb{R}$, and $\mathbb{C}$ stand for the set of natural numbers, integers, real numbers, and complex numbers, respectively. In order to facilitate the discussion below, we further introduce the following notations:

(i) $B C(\mathbb{R}, X)($ resp., $B C(\mathbb{R} \times Y, X)$ and $B C(\mathbb{R} \times Y \times Y, X))$ : the Banach space of bounded continuous functions from $\mathbb{R}$ to $X$ (resp., from $\mathbb{R} \times Y$ to $X$ and from $\mathbb{R} \times Y \times Y$ to $X$ ) with the supremum norm; 
(ii) $C(\mathbb{R}, X)$ (resp., $C(\mathbb{R} \times Y, X), C(\mathbb{R} \times Y \times Y, X))$ : the set of continuous functions from $\mathbb{R}$ to $X$ (resp., from $\mathbb{R} \times Y$ to $X$ and from $\mathbb{R} \times Y \times Y$ to $X)$;

(iii) $L(X, Y)$ : the Banach space of bounded linear operators from $X$ to $Y$ endowed with the operator topology. In particular, we write $L(X)$ when $X=Y$;

(iv) $L^{p}(\mathbb{R}, X)$ : the space of all classes of equivalence (with respect to the equality almost everywhere on $\mathbb{R}$ ) of measurable functions $f: \mathbb{R} \rightarrow X$ such that $\|f\| \epsilon$ $L^{p}(\mathbb{R}, \mathbb{R}) ;$

(v) $L_{\text {loc }}^{p}(\mathbb{R}, X)$ : standing for the space of all classes of equivalence of measurable functions $f: \mathbb{R} \rightarrow$ $X$ such that the restriction of $f$ to every bounded subinterval $I$ of $\mathbb{R}$ is in $L^{p}(I, X)$.

\subsection{Sectorial Operators and Riemann-Liouville \\ Fractional Derivative}

Definition 1 (see [13]). A closed and densely defined linear operator $A$ is said to be sectorial of type $\widetilde{\omega}$ if there exist $0<\theta<\pi / 2, M>0$, and $\widetilde{\omega} \in \mathbb{R}$ such that its resolvent exists outside the sector

$$
\begin{gathered}
\widetilde{\omega}+S_{\theta}:=\{\widetilde{\omega}+\lambda: \lambda \in \mathbb{C},|\arg (-\lambda)|<\theta\}, \\
\left\|(\lambda I-A)^{-1}\right\| \leq \frac{M}{|\lambda-\widetilde{\omega}|}, \quad \lambda \notin \widetilde{\omega}+S_{\theta} .
\end{gathered}
$$

The sectorial operators are well studied in the literature; we refer to [13] for more details.

Definition 2 (see [14]). Let $1<\alpha<2$ be given. Let $A$ be a closed and linear operator with domain $D(A)$ defined on a Banach space $X$. We call $A$ is the generator of a solution operator if there exist $\widetilde{\omega} \in \mathbb{R}$ and a strong continuous function $S_{\alpha}: \mathbb{R}^{+} \rightarrow L(X)$ such that $\left\{\lambda^{\alpha}: \operatorname{Re} \lambda>\widetilde{\omega}\right\} \subset \rho(A)$ and

$$
\begin{aligned}
\lambda^{\alpha-1}\left(\lambda^{\alpha}-A\right)^{-1} x= & \int_{0}^{\infty} e^{-\lambda t} S_{\alpha}(t) x d t \\
& \operatorname{Re} \lambda>\widetilde{\omega}, x \in X
\end{aligned}
$$

In this case, $S_{\alpha}(t)$ is called the solution operator generated by A.

Note that if $A$ is sectorial of type $\widetilde{\omega}$ with $0<\theta<\pi(1-\alpha / 2)$, then $A$ the generator of a solution operator given by

$$
S_{\alpha}(t):=\frac{1}{2 \pi i} \int_{\gamma} e^{\lambda t} \lambda^{\alpha-1}\left(\lambda^{\alpha}-A\right)^{-1} d \lambda,
$$

where $\gamma$ is a suitable path lying outside the sector $\widetilde{\omega}+S_{\theta}$ (see [15]). Recently, Cuesta [15] has proved that if $A$ is a sectorial operator of type $\widetilde{\omega}<0$ for some $0<\theta<\pi(1-\alpha / 2)(1<\alpha<$ 2), $M>0$, then there exists $C>0$ such that

$$
\left\|S_{\alpha}(t)\right\| \leq \frac{C M}{1+|\widetilde{\omega}| t^{\alpha}}, \quad t \geq 0 .
$$

Note that

$$
\int_{0}^{\infty} \frac{1}{1+|\widetilde{\omega}| t^{\alpha}} d t=\frac{|\widetilde{\omega}|^{-1 / \alpha} \pi}{\alpha \sin (\pi / \alpha)}
$$

for $1<\alpha<2$; therefore $S_{\alpha}(t)$ is integrable on $(0, \infty)$.

In the rest of this section, we list some necessary basic definitions in the theory of fractional calculus.

Definition 3 (see [16]). The fractional order integral of order $\alpha>0$ with the low limit $t_{0}>0$ for a function $f$ is defined as

$$
I^{\alpha} f(t)=\frac{1}{\Gamma(\alpha)} \int_{t_{0}}^{t}(t-s)^{\alpha-1} f(s) d s, \quad t>t_{0}, \alpha>0,
$$

provided the right-hand side is pointwise defined on $\left[t_{0}, \infty\right)$, where $\Gamma$ is the Gamma function.

Definition 4 (see [16]). Riemann-Liouville derivative of order $\alpha>0$ with the low limit $t_{0}>0$ for a function $f:\left[t_{0}, \infty\right) \rightarrow \mathbb{R}$ can be written as

$$
\begin{array}{r}
D_{t}^{\alpha} f(t)=\frac{1}{\Gamma(n-\alpha)} \frac{d^{n}}{d t^{n}} \int_{t_{0}}^{t}(t-s)^{n-\alpha-1} f(s) d s, \\
t>t_{0}, n-1<\alpha<n .
\end{array}
$$

\subsection{Evolution Family and Exponential Dichotomy}

Definition 5. A family of bounded linear operators $(U(t, s))_{t \geq s}$ on a Banach space $X$ is called a strong continuous evolution family if

(i) $U(t, r) U(r, s)=U(t, s)$ and $U(s, s)=I$ for all $t \geq r \geq s$ and $t, r, s \in \mathbb{R}$;

(ii) the map $(t, s) \rightarrow U(t, s) x$ is continuous for all $x \in X$, $t \geq s$ and $t, s \in \mathbb{R}$.

Definition 6. An evolution family $(U(t, s))_{t \geq s}$ on a Banach space $X$ is called hyperbolic (or has an exponential dichotomy) if there exist projections $P(t), t \in \mathbb{R}$, uniformly bounded and strong continuous in $t$, and constants $c>0$ and $\delta>0$ such that

(i) $U(t, s) P(s)=P(t) U(t, s)$ for $t \geq s$ and $t, s \in \mathbb{R}$;

(ii) the restriction $U_{\mathrm{Q}}(t, s): \mathrm{Q}(s) X \rightarrow \mathrm{Q}(t) X$ of $U(t, s)$ is invertible for $t \geq s$ (and set $U_{Q}(s, t):=U(t, s)^{-1}$ );

(iii)

$$
\begin{gathered}
\|U(t, s) P(s)\| \leq c e^{-\delta(t-s)}, \\
\left\|U_{Q}(s, t) Q(t)\right\| \leq c e^{-\delta(t-s)},
\end{gathered}
$$

for $t \geq s$ and $t, s \in \mathbb{R}$. Here and below we set $Q:=$ $I-P$.

Remark 7. Exponential dichotomy is a classical concept in the study of long-time behaviour of evolution equations. If $P(t)=$ $I$ for $t \in \mathbb{R}$, then $(U(t, s))_{t \geq s}$ is exponential stable. One can see [17-21] for more details. 
If $(U(t, s))_{t \geq s}$ is hyperbolic, then

$$
\Gamma(t, s):= \begin{cases}U(t, s) P(s), & t \geq s, t, s \in \mathbb{R}, \\ -U_{Q}(t, s) Q(s), & t<s, t, s \in \mathbb{R},\end{cases}
$$

is called Green's function corresponding to $(U(t, s))_{t \geq s}, P(\cdot)$ and

$$
\|\Gamma(t, s)\| \leq \begin{cases}c e^{-\delta(t-s)}, & t \geq s, t, s \in \mathbb{R} \\ c e^{-\delta(s-t)}, & t<s, t, s \in \mathbb{R}\end{cases}
$$

2.3. Weighted Pseudoperiodicity. First, let us recall some definitions of weighted pseudo anti-periodic function and weighted pseudoperiodic function.

Definition 8. A function $f \in C(\mathbb{R}, X)$ is said to be antiperiodic if there exists a $\omega \in \mathbb{R} \backslash\{0\}$ with the property that $f(t+\omega)=-f(t)$ for all $t \in \mathbb{R}$. If there exists a least positive $\omega$ with this property is called the anti-periodic of $f$. The collection of those functions is denoted by $P_{\omega a p}(\mathbb{R}, X)$.

Definition 9. A function $f \in C(\mathbb{R}, X)$ is said to be periodic if there exists a $\omega \in \mathbb{R} \backslash\{0\}$ with the property that $f(t+\omega)=f(t)$ for all $t \in \mathbb{R}$. If there exists a least positive $\omega$ with this property is called the periodic of $f$. The collection of those $\omega$-periodic functions is denoted by $P_{\omega}(\mathbb{R}, X)$.

Example 10. An example of a function that is anti-periodic is given by

$$
f(t)=\sum_{k=1}^{\infty} \frac{\sin [(2 k+1) t]}{k^{2}}, \quad t \in \mathbb{R},
$$

whose anti-periodic is $\pi$.

Other examples of nontrivial anti-periodic functions have been constructed in [22].

Remark 11. Note that if $f \in P_{\omega a p}(\mathbb{R}, X)$, then $f \in P_{2 \omega}(\mathbb{R}, X)$.

Definition 12. A function $f \in C(\mathbb{R} \times X, X)$ (resp., $C(\mathbb{R} \times X \times$ $X, X))$ is said to be periodic in $t \in \mathbb{R}$ uniform in $u \in X$ (resp., $(u, v) \in X \times X)$ if there exists a $\omega \in \mathbb{R} \backslash\{0\}$ with the property that $f(t+\omega, u)=f(t, u)$ for all $t \in \mathbb{R}, u \in X$ (resp., $f(t+\omega, u, v)=f(t, u, v)$ for all $t \in \mathbb{R},(u, v) \in X \times X)$. The collection of those $\omega$-periodic functions is denoted by $P_{\omega}(\mathbb{R} \times X, X)$ (resp., $\left.P_{\omega}(\mathbb{R} \times X \times X, X)\right)$.

$$
\begin{aligned}
& \text { Let } \\
& C_{0}(\mathbb{R}, X)=\left\{f \in B C(\mathbb{R}, X), \lim _{|t| \rightarrow \infty}\|f(t)\| d t=0\right\} .
\end{aligned}
$$

Definition 13 (see [23]). A function $f \in B C(\mathbb{R}, X)$ is said to be asymptotically anti-periodic if there exist $g \in P_{\omega a p}(\mathbb{R}, X)$ and $\varphi \in C_{0}(\mathbb{R}, X)$ such that $f=g+\varphi$. Denote by $A P_{\omega a p}(\mathbb{R}, X)$ the collection of such functions.

Definition 14 (see [24]). A function $f \in B C(\mathbb{R}, X)$ is said to be asymptotically periodic if there exist $g \in P_{\omega}(\mathbb{R}, X)$ and $\varphi \in C_{0}(\mathbb{R}, X)$ such that $f=g+\varphi$. Denote by $A P_{\omega}(\mathbb{R}, X)$ the collection of such functions.
Definition 15. A function $f \in B C(\mathbb{R}, X)$ is said to be a pseudo anti-periodic if it can be decomposed as $f=$ $g+\varphi$, where $g \in P_{\omega a p}(\mathbb{R}, X)$ and $\varphi \in B C(\mathbb{R}, X)$ with $\lim _{T \rightarrow \infty}(1 / 2 T) \int_{-T}^{T}\|\varphi(t)\| d t=0$. Denote by $P P_{\omega a p}(\mathbb{R}, X)$ the collection of such functions.

Definition 16 (see [9]). A function $f \in B C(\mathbb{R}, X)$ is said to be a pseudoperiodic if it can be decomposed as $f=$ $g+\varphi$, where $g \in P_{\omega}(\mathbb{R}, X)$ and $\varphi \in B C(\mathbb{R}, X)$ with $\lim _{T \rightarrow \infty}(1 / 2 T) \int_{-T}^{T}\|\varphi(t)\| d t=0$. Denote by $P P_{\omega}(\mathbb{R}, X)$ the collection of such functions.

Let $U$ be the set of all functions $\rho: \mathbb{R} \rightarrow(0, \infty)$ which are positive and locally integrable over $\mathbb{R}$. For a given $T>0$ and each $\rho \in U$, set

$$
\mu(T, \rho):=\int_{-T}^{T} \rho(t) d t .
$$

Define

$$
\begin{gathered}
U_{\infty}:=\left\{\rho \in U: \lim _{T \rightarrow \infty} \mu(T, \rho)=\infty\right\}, \\
U_{B}:=\left\{\rho \in U_{\infty}: \rho \text { is bounded and } \inf _{x \in \mathbb{R}} \rho(x)>0\right\} .
\end{gathered}
$$

It is clear that $U_{B} \subset U_{\infty} \subset U$.

Definition 17. Let $\rho_{1}, \rho_{2} \in U_{\infty} ; \rho_{1}$ is said to be equivalent to $\rho_{2}$ (i.e., $\left.\rho_{1} \sim \rho_{2}\right)$ if $\left(\rho_{1} / \rho_{2}\right) \in U_{B}$.

It is trivial to show that " $\sim$ " is a binary equivalence relation on $U_{\infty}$. The equivalence class of a given weight $\rho \in U_{\infty}$ is denoted by $\operatorname{cl}(\rho)=\left\{\varrho \in U_{\infty}: \rho \sim \varrho\right\}$. It is clear that $U_{\infty}=$ $\bigcup_{\rho \in U_{\infty}} \operatorname{cl}(\rho)$.

For $\rho_{1} \in U_{\infty}$, define the weighted ergodic space

$$
\begin{gathered}
W P P_{0}\left(\mathbb{R}, X, \rho_{1}\right):=\left\{f \in B C(\mathbb{R}, X): \lim _{T \rightarrow \infty} \frac{1}{\mu\left(T, \rho_{1}\right)}\right. \\
\left.\times \int_{-T}^{T} \rho_{1}(t)\|f(t)\| d t=0\right\} .
\end{gathered}
$$

Particularly, for $\rho_{1}, \rho_{2} \in U_{\infty}$, define [25]

$$
\begin{aligned}
W P P_{0}\left(\mathbb{R}, X, \rho_{1}, \rho_{2}\right):= & \left\{f \in B C(\mathbb{R}, X): \lim _{T \rightarrow \infty} \frac{1}{\mu\left(T, \rho_{1}\right)}\right. \\
& \left.\times \int_{-T}^{T} \rho_{2}(t)\|f(t)\| d t=0\right\} ;
\end{aligned}
$$

clearly, when $\rho_{1} \sim \rho_{2}$, this space coincide with $W P P_{0}(\mathbb{R}$, $\left.X, \rho_{1}\right)$; that is, $W P P_{0}\left(\mathbb{R}, X, \rho_{1}, \rho_{2}\right)=W P P_{0}\left(\mathbb{R}, X, \rho_{2}, \rho_{1}\right)=$ $W P P_{0}\left(\mathbb{R}, X, \rho_{1}\right)=W P P_{0}\left(\mathbb{R}, X, \rho_{2}\right)$; this fact suggests that $W P P_{0}\left(\mathbb{R}, X, \rho_{1}, \rho_{2}\right)$ are more interesting when $\rho_{1}$ and $\rho_{2}$ are not necessarily equivalent. So $W P P_{0}\left(\mathbb{R}, X, \rho_{1}, \rho_{2}\right)$ are general and richer than $W P P_{0}\left(\mathbb{R}, X, \rho_{1}\right)$ and give rise to an enlarged space of weighted pseudoperiodic function defined in [1]. 
Definition 18. Let $\rho_{1}, \rho_{2} \in U_{\infty}$. A function $f \in C(\mathbb{R}, X)$ is called weighted pseudo anti-periodic for $\omega \in \mathbb{R} \backslash\{0\}$ if it can be decomposed as $f=g+\varphi$, where $g \in P_{\text {wap }}(\mathbb{R}, X)$ and $\varphi \in W P P_{0}\left(\mathbb{R}, X, \rho_{1}, \rho_{2}\right)$. Denote by $W P P_{\omega a p}\left(\mathbb{R}, X, \rho_{1}, \rho_{2}\right)$ the set of such functions.

Definition 19. Let $\rho_{1}, \rho_{2} \in U_{\infty}$. A function $f \in C(\mathbb{R}, X)$ is called weighted pseudoperiodic for $\omega \in \mathbb{R} \backslash\{0\}$ if it can be decomposed as $f=g+\varphi$, where $g \in P_{\omega}(\mathbb{R}, X)$ and $\varphi \in$ $W P P_{0}\left(\mathbb{R}, X, \rho_{1}, \rho_{2}\right)$. Denote by $W P P_{\omega}\left(\mathbb{R}, X, \rho_{1}, \rho_{2}\right)$ the set of such functions.

Remark 20. If $\rho_{1} \sim \rho_{2}, W P P_{\omega a p}\left(\mathbb{R}, X, \rho_{1}, \rho_{2}\right)$ and $W P P_{\omega}(\mathbb{R}$, $X, \rho_{1}, \rho_{2}$ ) coincide with the weighted pseudo anti-periodic function and weighted pseudoperiodic function, respectively, introduced by [1].

Similarly, define

$$
\begin{aligned}
& W P P_{0}\left(\mathbb{R} \times X, X, \rho_{1}, \rho_{2}\right) \\
&:=\left\{f \in B C(\mathbb{R} \times X, X): \lim _{T \rightarrow \infty} \frac{1}{\mu\left(T, \rho_{1}\right)}\right. \\
& \times \int_{-T}^{T} \rho_{2}(t)\|f(t, u)\| d t=0 \\
& W P P_{0}(\left.\mathbb{R} \times X \times X, X, \rho_{1}, \rho_{2}\right) \\
&\text { uniformly in } u \in X\}, \\
& \quad=\left\{\begin{array}{c}
f \in(\mathbb{R} \times X \times X, X): \lim _{T \rightarrow \infty} \frac{1}{\mu\left(T, \rho_{1}\right)} \\
\times \int_{-T}^{T} \rho_{2}(t)\|f(t, u, v)\| d t=0 \\
\text { uniformly in }(u, v) \in X \times X\} .
\end{array}\right.
\end{aligned}
$$

Definition 21. Let $\rho_{1}, \rho_{2} \in U_{\infty}$. A function $f \in C(\mathbb{R} \times X, X)$ (resp., $C(\mathbb{R} \times X \times X, X)$ ) is called weighted pseudoperiodic in $t \in \mathbb{R}$ uniform in $u \in X$ (resp., $(u, v) \in X \times X)$ if it can be decomposed as $f=g+\varphi$, where $g \in P_{\omega}(\mathbb{R} \times X, X)$ (resp., $\left.P_{\omega}(\mathbb{R} \times X \times X, X)\right)$ and $\varphi \in W P P_{0}\left(\mathbb{R} \times X, X, \rho_{1}, \rho_{2}\right)$ (resp., $\left.W P P_{0}\left(\mathbb{R} \times X \times X, X, \rho_{1}, \rho_{2}\right)\right)$. Denote by $W P P_{\omega}\left(\mathbb{R} \times X, X, \rho_{1}, \rho_{2}\right)$ (resp., $\left.W P P_{\omega}\left(\mathbb{R} \times X \times X, X, \rho_{1}, \rho_{2}\right)\right)$ the set of such functions.

Next, we show some properties of the space $W P P_{\omega}(\mathbb{R}$, $\left.X, \rho_{1}, \rho_{2}\right)$. Similarly results are hold for $W P P_{\omega a p}\left(\mathbb{R}, X, \rho_{1}, \rho_{2}\right)$.

Lemma 22. Let $f \in B C(\mathbb{R}, X)$; then $f \in W P P_{0}\left(\mathbb{R}, X, \rho_{1}, \rho_{2}\right)$, $\rho_{1}, \rho_{2} \in U_{\infty}$, and $\sup _{T>0}\left(\mu\left(T, \rho_{2}\right) / \mu\left(T, \rho_{1}\right)\right)<\infty$ if and only if, for every $\varepsilon>0$,

$$
\lim _{T \rightarrow \infty} \frac{1}{\mu\left(T, \rho_{1}\right)} \int_{M(T, \varepsilon, f)} \rho_{2}(t) d t=0,
$$

where $M(T, \varepsilon, f):=\{t \in[-T, T]:\|f(t)\| \geq \varepsilon\}$.
Proof. The proof is similar to the one in [26].

Sufficiency. From the statement of the lemma it is clear that, for any $\varepsilon>0$, there exists $T_{0}>0$ such that, for $T>T_{0}$,

$$
\frac{1}{\mu\left(T, \rho_{1}\right)} \int_{M(T, \varepsilon, f)} \rho_{2}(t) d t<\frac{\varepsilon}{\|f\|} .
$$

Then

$$
\begin{aligned}
& \frac{1}{\mu\left(T, \rho_{1}\right)} \int_{-T}^{T} \rho_{2}(t)\|f(t)\| d t \\
& =\frac{1}{\mu\left(T, \rho_{1}\right)} \int_{M(T, \varepsilon, f)} \rho_{2}(t)\|f(t)\| d t \\
& \quad+\frac{1}{\mu\left(T, \rho_{1}\right)} \int_{[-T, T] \backslash M(T, \varepsilon, f)} \rho_{2}(t)\|f(t)\| d t \\
& \leq \frac{\|f\|}{\mu\left(T, \rho_{1}\right)} \int_{M(T, \varepsilon, f)} \rho_{2}(t) d t \\
& \quad+\frac{\varepsilon}{\mu\left(T, \rho_{1}\right)} \int_{-T}^{T} \rho_{2}(t) d t \\
& \leq \varepsilon+\sup _{T>0} \frac{\mu\left(T, \rho_{2}\right)}{\mu\left(T, \rho_{1}\right)} \varepsilon,
\end{aligned}
$$

so

$$
\lim _{T \rightarrow \infty} \frac{1}{\mu\left(T, \rho_{1}\right)} \int_{-T}^{T} \rho_{2}(t)\|f(t)\| d t=0 .
$$

That is, $f \in W P P_{0}\left(\mathbb{R}, X, \rho_{1}, \rho_{2}\right)$.

Necessity. Suppose the contrary, that there exists $\varepsilon_{0}>0$ such that

$$
\frac{1}{\mu\left(T, \rho_{1}\right)} \int_{M\left(T, \varepsilon_{0}, f\right)} \rho_{2}(t) d t
$$

does not converge to 0 as $T \rightarrow \infty$. Then there exists $\delta>0$ such that, for each $n$,

$$
\frac{1}{\mu\left(T_{n}, \rho_{1}\right)} \int_{M\left(T_{n}, \varepsilon_{0}, f\right)} \rho_{2}(t) d t \geq \delta \quad \text { for some } T_{n} \geq n \text {. }
$$

Then

$$
\begin{aligned}
& \frac{1}{\mu\left(T_{n}, \rho_{1}\right)} \int_{-T_{n}}^{T_{n}} \rho_{2}(t)\|f(t)\| d t \\
& \quad \geq \frac{1}{\mu\left(T_{n}, \rho_{1}\right)} \int_{M\left(T_{n}, \varepsilon_{0}, f\right)} \rho_{2}(t)\|f(t)\| d t \\
& \geq \frac{\varepsilon_{0}}{\mu\left(T_{n}, \rho_{1}\right)} \int_{M\left(T_{n}, \varepsilon_{0}, f\right)} \rho_{2}(t) d t \\
& \geq \varepsilon_{0} \delta,
\end{aligned}
$$

which contradicts the fact that $f \in W P P_{0}\left(\mathbb{R}, X, \rho_{1}, \rho_{2}\right)$, and the proof is complete. 
Let $W P P_{0}\left(\mathbb{R}, \mathbb{R}^{+}, \rho_{1}, \rho_{2}\right)=\left\{f \in W P P_{0}\left(\mathbb{R}, \mathbb{R}, \rho_{1}, \rho_{2}\right)\right.$ : $f(t) \geq 0$, for all $t \in \mathbb{R}\}$.

Lemma 23. Let $\alpha>0$; then $f \in W P P_{0}\left(\mathbb{R}, \mathbb{R}^{+}, \rho_{1}, \rho_{2}\right)$ if and only if $f^{\alpha} \in W P P_{0}\left(\mathbb{R}, \mathbb{R}^{+}, \rho_{1}, \rho_{2}\right)$, where $f^{\alpha}(t)=[f(t)]^{\alpha}$, $\rho_{1}, \rho_{2} \in U_{\infty}$, and $\sup _{T>0}\left(\mu\left(T, \rho_{2}\right) / \mu\left(T, \rho_{1}\right)\right)<\infty$.

Proof. By Lemma 22, $f \in W P P_{0}\left(\mathbb{R}, \mathbb{R}^{+}, \rho_{1}, \rho_{2}\right)$ if and only if, for every $\varepsilon>0$,

$$
\lim _{T \rightarrow \infty} \frac{1}{\mu\left(T, \rho_{1}\right)} \int_{M(T, \varepsilon, f)} \rho_{2}(t) d t=0,
$$

where $M(T, \varepsilon, f):=\{t \in[-T, T]: f(t) \geq \varepsilon\}$. It is equivalent to for every $\varepsilon>0$,

$$
\lim _{T \rightarrow \infty} \frac{1}{\mu\left(T, \rho_{1}\right)} \int_{M\left(T, \varepsilon, f^{\alpha}\right)} \rho_{2}(t) d t=0,
$$

where $M\left(T, \varepsilon, f^{\alpha}\right):=\left\{t \in[-T, T]: f^{\alpha}(t) \geq \varepsilon\right\}$. So $f^{\alpha} \in$ $W P P_{0}\left(\mathbb{R}, \mathbb{R}^{+}, \rho_{1}, \rho_{2}\right)$.

Lemma 24. Let $\varphi_{n} \rightarrow \varphi$ uniformly on $\mathbb{R}$ where each $\varphi_{n} \in$ $W P P_{0}\left(\mathbb{R}, X, \rho_{1}, \rho_{2}\right)$; if $\sup _{T>0}\left(\mu\left(T, \rho_{2}\right) / \mu\left(T, \rho_{1}\right)\right)<\infty$, then $\varphi \in W P P_{0}\left(\mathbb{R}, X, \rho_{1}, \rho_{2}\right)$.

Proof. For $T>0$,

$$
\begin{aligned}
& \frac{1}{\mu\left(T, \rho_{1}\right)} \int_{-T}^{T} \rho_{2}(t)\|\varphi(t)\| d t \\
& \leq \frac{1}{\mu\left(T, \rho_{1}\right)} \int_{-T}^{T} \rho_{2}(t)\left\|\varphi_{n}(t)-\varphi(t)\right\| d t \\
&+\frac{1}{\mu\left(T, \rho_{1}\right)} \int_{-T}^{T} \rho_{2}(t)\left\|\varphi_{n}(t)\right\| d t \\
& \leq \frac{\mu\left(T, \rho_{2}\right)}{\mu\left(T, \rho_{1}\right)}\left\|\varphi_{n}-\varphi\right\| \\
&+\frac{1}{\mu\left(T, \rho_{1}\right)} \int_{-T}^{T} \rho_{2}(t)\left\|\varphi_{n}(t)\right\| d t \\
& \leq \sup _{T>0} \frac{\mu\left(T, \rho_{2}\right)}{\mu\left(T, \rho_{1}\right)}\left\|\varphi_{n}-\varphi\right\| \\
&+\frac{1}{\mu\left(T, \rho_{1}\right)} \int_{-T}^{T} \rho_{2}(t)\left\|\varphi_{n}(t)\right\| d t .
\end{aligned}
$$

Let $T \rightarrow \infty$ and then $n \rightarrow \infty$ in the above inequality; it follows that $\varphi \in W P P_{0}\left(\mathbb{R}, X, \rho_{1}, \rho_{2}\right)$.

Let $\rho \in U_{\infty}, \tau \in \mathbb{R}$, and define $\rho^{\tau}$ by $\rho^{\tau}(t)=\rho(t+\tau)$ for $t \in \mathbb{R}$. Define [27]

$$
U_{T}=\left\{\rho \in U_{\infty}: \rho \sim \rho^{\tau} \text { for each } \tau \in \mathbb{R}\right\} .
$$

It is easy to see that $U_{T}$ contains many of weights, such as 1 , $\left(1+t^{2}\right) /\left(2+t^{2}\right), e^{t}$, and $1+|t|^{n}$ with $n \in \mathbb{N}$ et al.

Lemma 25. Let $\rho_{1}, \rho_{2}, q_{1}, q_{2} \in U_{\infty}$ such that $\rho_{1} \sim q_{1}, \rho_{2} \sim q_{2}$; then $W P P_{0}\left(\mathbb{R}, X, \rho_{1}, \rho_{2}\right)=W P P_{0}\left(\mathbb{R}, X, q_{1}, q_{2}\right)$.
Proof. Since $\rho_{1} \sim q_{1}$ and $\rho_{2} \sim q_{2}$, there exist positive constants $K_{1}, K_{1}^{\prime}, K_{2}$, and $K_{2}^{\prime}$ such that $K_{1} q_{1} \leq \rho_{1} \leq K_{1}^{\prime} q_{1}$ and $K_{2} q_{2} \leq \rho_{2} \leq K_{2}^{\prime} q_{2}$; then,

$$
\begin{aligned}
& K_{1} \mu\left(T, q_{1}\right) \leq \mu\left(T, \rho_{1}\right) \leq K_{1}^{\prime} \mu\left(T, q_{1}\right), \\
& K_{2} \mu\left(T, q_{2}\right) \leq \mu\left(T, \rho_{2}\right) \leq K_{2}^{\prime} \mu\left(T, q_{2}\right) .
\end{aligned}
$$

Let $\varphi \in W P P_{0}\left(\mathbb{R}, X, q_{1}, q_{2}\right)$; then

$$
\begin{aligned}
& \frac{1}{\mu\left(T, \rho_{1}\right)} \int_{-T}^{T} \rho_{2}(t)\|\varphi(t)\| d t \\
& \quad=\frac{1}{\mu\left(T, \rho_{1}\right)} \int_{-T}^{T} \frac{\rho_{2}(t)}{q_{2}(t)} q_{2}(t)\|\varphi(t)\| d t \\
& \quad \leq \frac{K_{2}^{\prime}}{\mu\left(T, \rho_{1}\right)} \int_{-T}^{T} q_{2}(t)\|\varphi(t)\| d t \\
& \quad \leq \frac{K_{2}^{\prime}}{K_{1} \mu\left(T, q_{1}\right)} \int_{-T}^{T} q_{2}(t)\|\varphi(t)\| d t .
\end{aligned}
$$

$\varphi \in W P P_{0}\left(\mathbb{R}, X, q_{1}, q_{2}\right)$ implies that

$$
\lim _{T \rightarrow \infty} \frac{1}{\mu\left(T, q_{1}\right)} \int_{-T}^{T} q_{2}(t)\|\varphi(t)\| d t=0 ;
$$

then

$$
\lim _{T \rightarrow \infty} \frac{1}{\mu\left(T, \rho_{1}\right)} \int_{-T}^{T} \rho_{2}(t)\|\varphi(t)\| d t=0 .
$$

That is, $\varphi \in W P P_{0}\left(\mathbb{R}, X, \rho_{1}, \rho_{2}\right)$; hence

$$
W P P_{0}\left(\mathbb{R}, X, q_{1}, q_{2}\right) \subset W P P_{0}\left(\mathbb{R}, X, \rho_{1}, \rho_{2}\right) .
$$

Proceeding in a similar manner, we have $W P P_{0}\left(\mathbb{R}, X, \rho_{1}\right.$, $\left.\rho_{2}\right) \subset W P P_{0}\left(\mathbb{R}, X, q_{1}, q_{2}\right)$. The proof is complete.

Lemma 26. Let $\rho_{1}, \rho_{2} \in U_{T}$ and $\varphi \in W P P_{0}\left(\mathbb{R}, X, \rho_{1}, \rho_{2}\right)$; then $\varphi(\cdot-\tau) \in W P P_{0}\left(\mathbb{R}, X, \rho_{1}, \rho_{2}\right)$ for $\tau \in \mathbb{R}$.

Proof. Let $\varphi \in W P P_{0}\left(\mathbb{R}, X, \rho_{1}, \rho_{2}\right)$ and $\rho_{1}, \rho_{2} \in U_{T}$; by Lemma $25, \varphi \in W P P_{0}\left(\mathbb{R}, X, \rho_{1}^{\tau}, \rho_{2}^{\tau}\right)$ for $\tau \in \mathbb{R}$. Without loss of generality, we assume that $\tau>0$; then

$$
\begin{aligned}
& \frac{1}{\mu\left(T, \rho_{1}\right)} \int_{-T}^{T}\|\varphi(t-\tau)\| \rho_{2}(t) d t \\
& \quad=\frac{1}{\mu\left(T, \rho_{1}\right)} \int_{-T-\tau}^{T-\tau}\|\varphi(t)\| \rho_{2}(t+\tau) d t \\
& \quad \leq \frac{1}{\mu\left(T, \rho_{1}\right)} \int_{-T-\tau}^{T+\tau}\|\varphi(t)\| \rho_{2}^{\tau}(t) d t \\
& \quad=\frac{\mu\left(T+\tau, \rho_{1}^{\tau}\right)}{\mu\left(T, \rho_{1}\right) \mu\left(T+\tau, \rho_{1}^{\tau}\right)} \int_{-T-\tau}^{T+\tau}\|\varphi(t)\| \rho_{2}^{\tau}(t) d t .
\end{aligned}
$$


Since $\rho_{1} \in U_{T}$ implies that $\rho_{1} \sim \rho_{1}^{2 \tau}$, there exists $\eta>0$ such that $\rho_{1}^{2 \tau}(t) / \rho_{1}(t) \leq \eta$ for $t \in \mathbb{R}$. Then, for $T>\tau$,

$$
\begin{aligned}
\mu\left(T+\tau, \rho_{1}^{\tau}\right)= & \int_{-T-\tau}^{T+\tau} \rho_{1}^{\tau}(t) d t \\
= & \int_{-T}^{T+2 \tau} \rho_{1}(t) d t=\mu\left(T, \rho_{1}\right)+\int_{T}^{T+2 \tau} \rho_{1}(t) d t \\
= & \mu\left(T, \rho_{1}\right)+\int_{T-2 \tau}^{T} \rho_{1}^{2 \tau}(t) d t \leq \mu\left(T, \rho_{1}\right) \\
& +\int_{-T}^{T} \rho_{1}^{2 \tau}(t) d t \leq \mu\left(T, \rho_{1}\right) \\
& +\int_{-T}^{T} \eta \rho_{1}(t) d t=(\eta+1) \mu\left(T, \rho_{1}\right)
\end{aligned}
$$

Therefore, by $\varphi \in W P P_{0}\left(\mathbb{R}, X, \rho_{1}^{\tau}, \rho_{2}^{\tau}\right)$,

$$
\begin{array}{r}
\frac{1}{\mu\left(T, \rho_{1}\right)} \int_{-T}^{T}\|\varphi(t-\tau)\| \rho_{2}(t) d t \\
\leq \frac{\eta+1}{\mu\left(T+\tau, \rho_{1}^{\tau}\right)} \int_{-T-\tau}^{T+\tau}\|\varphi(t)\| \rho_{2}^{\tau}(t) d t \rightarrow 0 \\
\text { as } T \longrightarrow \infty,
\end{array}
$$

which implies that $\varphi(\cdot-\tau) \in W P P_{0}\left(\mathbb{R}, X, \rho_{1}, \rho_{2}\right)$. The proof is complete.

Using similar ideas as in $[25,28]$, one can easily show the following result.

Lemma 27. If $\rho_{1}, \rho_{2} \in U_{T}$ and $\inf _{T>0}\left(\mu\left(T, \rho_{2}\right) / \mu\left(T, \rho_{1}\right)\right)=$ $\delta_{0}>0$, then the decomposition of weighted pseudoperiodic function is unique.

By Lemma 27, it is obvious that $\left(W P P_{\omega}\left(\mathbb{R}, X, \rho_{1}, \rho_{2}\right),\|\cdot\|\right)$ (resp., $\left.\left(W P P_{\omega}\left(\mathbb{R} \times X, X, \rho_{1}, \rho_{2}\right),\|\cdot\|\right)\right), \rho_{1}, \rho_{2} \in U_{T}$, and $\inf _{T>0}\left(\mu\left(T, \rho_{2}\right) / \mu\left(T, \rho_{1}\right)\right)=\delta_{0}>0$ is a Banach space when endowed with the sup norm.

2.4. Weighted Stepanov-Like Pseudoperiodicity. In this subsection, we introduce the new class of functions called weighted $S^{p}$-pseudo anti-periodic function and weighted $S^{p}$. pseudoperiodic function and investigate the properties of these functions.

Let $p \in[1, \infty)$. The space $B S^{p}(\mathbb{R}, X)$ of all Stepanov bounded functions, with the exponent $p$, consists of all measurable functions $f: \mathbb{R} \rightarrow X$ such that $f^{b} \in L^{\infty}(\mathbb{R}$, $\left.L^{p}([0,1] ; X)\right)$, where $f^{b}$ is the Bochner transform of $f$ defined by $f^{b}(t, s):=f(t+s), t \in \mathbb{R}, s \in[0,1]$. BS $S^{p}(\mathbb{R}, X)$ is a Banach space with the norm [29]

$$
\|f\|_{S^{p}}=\left\|f^{b}\right\|_{L^{\infty}\left(\mathbb{R}, L^{p}\right)}=\sup _{t \in \mathbb{R}}\left(\int_{t}^{t+1}\|f(\tau)\|^{p} d \tau\right)^{1 / p}
$$

It is obvious that $L^{p}(\mathbb{R}, X) \subset B S^{p}(\mathbb{R}, X) \subset L_{\text {loc }}^{p}(\mathbb{R}, X)$ and $B S^{p}(\mathbb{R}, X) \subset B S^{q}(\mathbb{R}, X)$ for $p \geq q \geq 1$.

For $\rho_{1}, \rho_{2} \in U_{\infty}$, define the weighted ergodic space in $B S^{p}(\mathbb{R}, X)$ :

$$
\begin{aligned}
S^{p} W P P_{0} & \left(\mathbb{R}, X, \rho_{1}, \rho_{2}\right) \\
:= & \left\{f \in B S^{p}(\mathbb{R}, X): \lim _{T \rightarrow \infty} \frac{1}{\mu\left(T, \rho_{1}\right)}\right. \\
& \left.\times \int_{-T}^{T} \rho_{2}(t)\left(\int_{t}^{t+1}\|f(\sigma)\|^{p} d \sigma\right)^{1 / p} d t=0\right\} .
\end{aligned}
$$

Remark 28. It is clear that $f \in S^{p} W P P_{0}\left(\mathbb{R}, X, \rho_{1}, \rho_{2}\right)$ if and only if $f^{b} \in W P P_{0}\left(\mathbb{R}, L^{p}([0,1], X), \rho_{1}, \rho_{2}\right)$.

Definition 29. Let $\rho_{1}, \rho_{2} \in U_{\infty}$. A function $f \in B S^{p}(\mathbb{R}, X)$ is said to be weighted Stepanov-like pseudo anti-periodic (or weighted $S^{p}$-pseudo anti-periodic) if there is $\varphi \in$ $S^{p} W P P_{0}\left(\mathbb{R}, X, \rho_{1}, \rho_{2}\right)$ such that the function $g=f-\varphi$ satisfies $g(t+\omega)+g(t)=0$ a.e. $t \in \mathbb{R}$. Denote by $S^{p} W P P_{\omega a p}\left(\mathbb{R}, X, \rho_{1}, \rho_{2}\right)$ the collection of such functions.

Definition 30. Let $\rho_{1}, \rho_{2} \in U_{\infty}$. A function $f \in B S^{p}(\mathbb{R}$, $X$ ) is said to be weighted Stepanov-like pseudoperiodic (or weighted $S^{p}$-pseudoperiodic) if there is $\varphi \in S^{p} W P P_{0}(\mathbb{R}$, $\left.X, \rho_{1}, \rho_{2}\right)$ such that the function $g=f-\varphi$ satisfies $g(t+$ $\omega)-g(t)=0$ a.e. $t \in \mathbb{R}$. Denote by $S^{p} W P P_{\omega}\left(\mathbb{R}, X, \rho_{1}, \rho_{2}\right)$ the collection of such functions.

Remark 31. Let $\varphi$ be defined on $\mathbb{R}$ by

$$
\varphi(t)= \begin{cases}1, & t \in\left[2^{n}-1,2^{n}+1\right], n \in \mathbb{N}, \\ 0, & \text { otherwise. }\end{cases}
$$

For each $T>0$, denote $n_{T}=\left[\log _{2} T\right]+1$, where $[\cdot]$ is largest integer function; then

$$
2^{n_{T}-1} \leq T \leq 2^{n_{T}}
$$

Let $\rho_{1}=e^{t}$ and $\rho_{2}=1$; by a direct calculation, one has

$$
\begin{gathered}
\frac{1}{\mu\left(T, \rho_{1}\right)} \int_{-T}^{T} \rho_{2}(t)\left(\int_{t}^{t+1}\|\varphi(\sigma)\|^{p} d \sigma\right)^{1 / p} d t \\
\quad=\frac{1}{\mu\left(T, e^{t}\right)} \int_{-T}^{T}\left(\int_{t}^{t+1}\|\varphi(\sigma)\|^{p} d \sigma\right)^{1 / p} d t \\
\leq \frac{1}{\mu\left(T, e^{t}\right)} \int_{-2^{n_{T}}}^{2^{n_{T}}}\left(\int_{0}^{1}\|\varphi(\sigma+t)\|^{p} d \sigma\right)^{1 / p} d t \\
\leq \frac{3 n_{T}}{e^{T}-e^{-T}} \longrightarrow 0, \quad T \longrightarrow \infty .
\end{gathered}
$$

So $\varphi \in S^{p} W P P_{0}\left(\mathbb{R}, \mathbb{R}, \rho_{1}, \rho_{2}\right)$. In addition, it is obvious that $\varphi \notin W P P_{0}\left(\mathbb{R}, \mathbb{R}, \rho_{1}, \rho_{2}\right)$.

Let $g(t)=\sin t, f(t)=g(t)+\varphi(t)$; then $f \in S^{p} W P P_{2 \pi}(\mathbb{R}$, $\left.\mathbb{R}, \rho_{1}, \rho_{2}\right)$, but $f \notin W P P_{2 \pi}\left(\mathbb{R}, \mathbb{R}, \rho_{1}, \rho_{2}\right)$. 


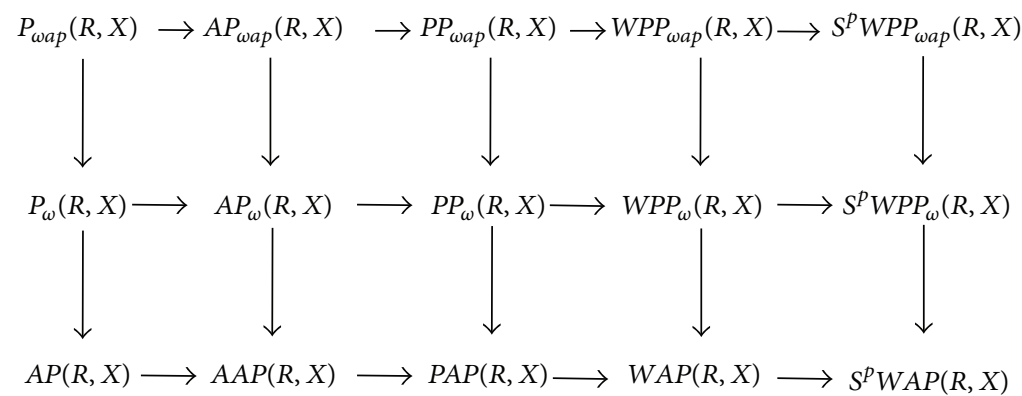

FIGURE 1: Relationship between anti-periodic, periodic or almost periodic functions and their extensions, where " $\rightarrow$ " denotes subset relation "C".

From definition, we have the following diagram that summarizes the different classes of subspaces defined in Figure 1 , where $A P(\mathbb{R}, X), A A P(\mathbb{R}, X)$, and so forth. stand for almost periodic and asymptotically almost periodic function, respectively; one can see [6] for more details, where the paper gives the historical development of almost periodicity and almost automorphy and relationship between these functions and their extensions.

Similarly, define

$$
\begin{aligned}
S^{p} W P_{0} & \left(\mathbb{R} \times X, X, \rho_{1}, \rho_{2}\right) \\
:= & \left\{f \in B S^{p}(\mathbb{R} \times X, X): \lim _{T \rightarrow \infty} \frac{1}{\mu\left(T, \rho_{1}\right)}\right. \\
& \times \int_{-T}^{T} \rho_{2}(t)\left(\int_{t}^{t+1}\|f(\sigma, u)\|^{p} d \sigma\right)^{1 / p} d t=0 \\
& \text { uniformly in } u \in X\}, \\
S^{p} W P P_{0}( & \left.\mathbb{R} \times X \times X, X, \rho_{1}, \rho_{2}\right) \\
:= & \left\{f \in B S^{p}(\mathbb{R} \times X \times X, X):\right. \\
& \lim _{T \rightarrow \infty} \frac{1}{\mu\left(T, \rho_{1}\right)} \int_{-T}^{T} \rho_{2}(t) \\
& \times\left(\int_{t}^{t+1}\|f(\sigma, u, v)\|^{p} d \sigma\right)^{1 / p} d t=0 \\
& \text { uniformly in }(u, v) \in X \times X\} .
\end{aligned}
$$

Definition 32. Let $\rho_{1}, \rho_{2} \in U_{\infty}$. A function $f \in B S^{p}(\mathbb{R} \times$ $X, X)$ (resp., $B S^{p}(\mathbb{R} \times X \times X, X)$ ) is called weighted $S^{p}$. pseudoperiodic in $t \in \mathbb{R}$ uniform in $u \in X$ (resp., $(u, v) \in$ $X \times X)$ if there is $\varphi \in S^{p} W P P_{0}\left(\mathbb{R} \times X, X, \rho_{1}, \rho_{2}\right)$ (resp., $\left.S^{p} W P P_{0}\left(\mathbb{R} \times X \times X, X, \rho_{1}, \rho_{2}\right)\right)$ such that the function $g=f-\varphi$ satisfies $g(t+\omega, u)-g(t, u)=0$ a.e. $t \in \mathbb{R}$ and each $u \in X$ (resp., $g(t+\omega, u, v)-g(t, u, v)=0$ a.e. $t \in \mathbb{R}$ and each $(u, v) \in X \times X)$. Denote by $S^{p} W P P_{\omega}\left(\mathbb{R} \times X, X, \rho_{1}, \rho_{2}\right)$ (resp., $\left.S^{p} W P P_{\omega}\left(\mathbb{R} \times X \times X, X, \rho_{1}, \rho_{2}\right)\right)$ the collection of such functions.
Next, we show some properties of the space $S^{p} W P P_{\omega}(\mathbb{R}$, $\left.X, \rho_{1}, \rho_{2}\right)$. Similarly results hold for $S^{p} W P P_{\omega a p}\left(\mathbb{R}, X, \rho_{1}, \rho_{2}\right)$.

Lemma 33. $W P P_{\omega}\left(\mathbb{R}, X, \rho_{1}, \rho_{2}\right) \subset S^{p} W P P_{\omega}\left(\mathbb{R}, X, \rho_{1}, \rho_{2}\right)$ for each $1 \leq p<\infty, \rho_{1}, \rho_{2} \in U_{T}$, $\sup _{T>0}\left(\mu\left(T, \rho_{2}\right) / \mu\left(T, \rho_{1}\right)\right)<\infty$.

Proof. If $f \in W P P_{\omega}\left(\mathbb{R}, X, \rho_{1}, \rho_{2}\right)$, let $f=f_{1}+f_{2}$, where $f_{1} \in P_{\omega}(\mathbb{R}, X)$ and $f_{2} \in W P P_{0}\left(\mathbb{R}, X, \rho_{1}, \rho_{2}\right)$. Then $\left\|f_{2}(\cdot)\right\| \in W P P_{0}\left(\mathbb{R}, \mathbb{R}^{+}, \rho_{1}, \rho_{2}\right)$. By Lemma $23,\left\|f_{2}(\cdot)\right\|^{p} \in$ $W P P_{0}\left(\mathbb{R}, \mathbb{R}^{+}, \rho_{1}, \rho_{2}\right)$. Note that $\left\|f_{2}(\cdot+\sigma)\right\|^{p} \in W P P_{0}(\mathbb{R}$, $\left.\mathbb{R}^{+}, \rho_{1}, \rho_{2}\right)$ for each $\sigma \in[0,1]$; by Lebesgue's dominated convergence theorem, one has

$$
\begin{array}{r}
\int_{0}^{1}\left(\frac{1}{\mu\left(T, \rho_{1}\right)} \int_{-T}^{T} \rho_{2}(t)\left\|f_{2}(t+\sigma)\right\|^{p} d t\right) d \sigma \\
T \longrightarrow 0 \\
T \longrightarrow \infty
\end{array}
$$

that is,

$$
\begin{array}{r}
\frac{1}{\mu\left(T, \rho_{1}\right)} \int_{-T}^{T} \rho_{2}(t)\left(\int_{0}^{1}\left\|f_{2}(t+\sigma)\right\|^{p} d \sigma\right) d t \\
\longrightarrow 0 \\
T \longrightarrow \infty
\end{array}
$$

which means that $h_{2} \in W P P_{0}\left(\mathbb{R}, \mathbb{R}^{+}, \rho_{1}, \rho_{2}\right)$, where

$$
h_{2}(t)=\int_{0}^{1}\left\|f_{2}(t+\sigma)\right\|^{p} d \sigma, \quad t \in \mathbb{R} .
$$

By Lemma $23, h_{2}^{1 / p} \in W P P_{0}\left(\mathbb{R}, \mathbb{R}^{+}, \rho_{1}, \rho_{2}\right)$; that is,

$$
\begin{array}{r}
\frac{1}{\mu\left(T, \rho_{1}\right)} \int_{-T}^{T} \rho_{2}(t)\left(\int_{0}^{1}\left\|f_{2}(t+\sigma)\right\|^{p} d \sigma\right)^{1 / p} d t \\
\longrightarrow 0, \\
T \longrightarrow \infty
\end{array}
$$

which means that $f_{2} \in S^{p} W P P_{0}\left(\mathbb{R}, X, \rho_{1}, \rho_{2}\right)$; then $f \in$ $S^{p} W P P_{\omega}\left(\mathbb{R}, X, \rho_{1}, \rho_{2}\right)$. The proof is complete.

Theorem 34. Assume that $\rho_{1}, \rho_{2} \in U_{\infty}, f=f_{1}+f_{2} \epsilon$ $S^{p} W P P_{\omega}\left(\mathbb{R} \times X \times X, X, \rho_{1}, \rho_{2}\right)$ with $f_{2} \in S^{p} W P P_{0}(\mathbb{R} \times X \times$ $\left.X, X, \rho_{1}, \rho_{2}\right), f_{1}(t+\omega, u, v)-f_{1}(t, u, v)=0$ a.e. $t \in \mathbb{R}$, 
$(u, v) \in X \times X$, and there exist constants $L_{f}, L_{f_{1}}>0$ such that

$$
\begin{gathered}
\left\|f\left(t, u_{1}, u_{2}\right)-f\left(t, v_{1}, v_{2}\right)\right\| \leq L_{f}\left(\left\|u_{1}-v_{1}\right\|+\left\|u_{2}-v_{2}\right\|\right), \\
t \in \mathbb{R}, u_{1}, u_{2}, v_{1}, v_{2} \in X, \\
\left\|f_{1}\left(t, u_{1}, u_{2}\right)-f_{1}\left(t, v_{1}, v_{2}\right)\right\| \\
\leq L_{f_{1}}\left(\left\|u_{1}-v_{1}\right\|+\left\|u_{2}-v_{2}\right\|\right) \\
t \in \mathbb{R}, u_{1}, u_{2}, v_{1}, v_{2} \in X ;
\end{gathered}
$$

then $f\left(\cdot, h_{1}(\cdot), h_{2}(\cdot)\right) \in S^{p} W P P_{\omega}\left(\mathbb{R}, X, \rho_{1}, \rho_{2}\right)$ if $h_{1}, h_{2} \in$ $S^{p} W P P_{\omega}\left(\mathbb{R}, X, \rho_{1}, \rho_{2}\right)$.

The proof is similar to that of Theorem 3.6 in [6] and the details are omitted here.

Remark 35. It is not difficult to see that Theorem 34 holds for $S^{p} W P P_{\omega a p}\left(\mathbb{R}, X, \rho_{1}, \rho_{2}\right)$.

Lemma 36. Let $\{S(t)\}_{t \geq 0} \subset L(X)$ be a strongly continuous family of bounded and linear operators such that $\|S(t)\| \leq$ $\phi(t), t \in \mathbb{R}^{+}$, where $\phi \in L^{1}\left(\mathbb{R}^{+}\right)$is nonincreasing. If $f \in S^{p} W P P_{\omega}\left(\mathbb{R}, X, \rho_{1}, \rho_{2}\right), \rho_{1}, \rho_{2} \in U_{\infty}, \inf _{T>0}(\mu(T$, $\left.\left.\rho_{2}\right) / \mu\left(T, \rho_{1}\right)\right)=\delta_{0}>0$, and $\sup _{T>0}\left(\mu\left(T, \rho_{2}\right) / \mu\left(T, \rho_{1}\right)\right)<\infty$; then

$$
(\Lambda f)(t):=\int_{-\infty}^{t} S(t-s) f(s) d s \in W P P_{\omega}\left(\mathbb{R}, X, \rho_{1}, \rho_{2}\right)
$$

Proof. Let $f(t)=f_{1}(t)+f_{2}(t)$, where $f_{2} \in S^{p} W P P_{0}(\mathbb{R}$, $\left.X, \rho_{1}, \rho_{2}\right)$ and $f_{1}(t+\omega)-f_{1}(t)=0$ a.e. $t \in \mathbb{R}$, then

$$
\begin{aligned}
(\Lambda f)(t) & =\int_{-\infty}^{t} S(t-s) f_{1}(s) d s+\int_{-\infty}^{t} S(t-s) f_{2}(s) d s \\
& :=\left(\Lambda_{1} f\right)(t)+\left(\Lambda_{2} f\right)(t) .
\end{aligned}
$$

First, we show that $\Lambda_{2} f \in W P P_{0}\left(\mathbb{R}, X, \rho_{1}, \rho_{2}\right)$. Consider the integrals

$$
Y_{n}(t)=\int_{t-n}^{t-n+1} S(t-s) f_{2}(s) d s
$$

For each $n \in \mathbb{N}$, by the principle of uniform boundedness,

$$
L_{n}:=\sup _{n-1 \leq t \leq n} S(t)<\infty
$$

Fix $n \in \mathbb{N}$ and $t \in \mathbb{R}$; we have

$$
\begin{aligned}
\left\|Y_{n}(t+h)-Y_{n}(t)\right\| & \leq \int_{n-1}^{n} S(s)\left\|f_{2}(t+h-s)-f_{2}(t-s)\right\| d s \\
& \leq L_{n} \int_{t-n}^{t-n+1}\left\|f_{2}(s+h)-f_{2}(s)\right\| d s \\
& \leq L_{n}\left(\int_{t-n}^{t-n+1}\left\|f_{2}(s+h)-f_{2}(s)\right\|^{p} d s\right)^{1 / p} .
\end{aligned}
$$

In view of $f_{2} \in L_{\text {loc }}^{p}(\mathbb{R}, X)$, we get

$$
\lim _{h \rightarrow 0} \int_{t-n}^{t-n+1}\left\|f_{2}(s+h)-f_{2}(s)\right\|^{p} d s=0
$$

which yields that

$$
\lim _{h \rightarrow 0}\left\|Y_{n}(t+h)-Y_{n}(t)\right\|=0
$$

This means that $Y_{n}(t)$ is continuous.

By Hölder inequality, one has

$$
\begin{aligned}
\left\|Y_{n}(t)\right\| & \leq \int_{n-1}^{n}\left\|S(s) f_{2}(t-s)\right\| d s \\
& \leq \int_{n-1}^{n} \phi(s)\left\|f_{2}(t-s)\right\| d s \\
& \leq \phi(n-1) \int_{n-1}^{n}\left\|f_{2}(t-s)\right\| d s \\
& \leq \phi(n-1) \int_{t-n}^{t-n+1}\left\|f_{2}(s)\right\| d s \\
& \leq \phi(n-1)\left(\int_{t-n}^{t-n+1}\left\|f_{2}(s)\right\|^{p} d s\right)^{1 / p} \\
& \leq \phi(n-1)\left\|f_{2}\right\|_{S^{p}},
\end{aligned}
$$

since

$$
\begin{aligned}
\sum_{n=1}^{\infty} \phi(n-1)\left\|f_{2}\right\|_{S^{p}} & \leq\left(\phi(0)+\sum_{n=2}^{\infty} \int_{n-2}^{n-1} \phi(t) d t\right)\left\|f_{2}\right\|_{S^{p}} \\
& \leq\left(\phi(0)+\|\phi\|_{L^{1}}\right)\left\|f_{2}\right\|_{S^{p}}<\infty
\end{aligned}
$$

then $\sum_{n=1}^{\infty} Y_{n}(t)$ is uniform convergent on $\mathbb{R}$. Let $Y(t)=$ $\sum_{n=1}^{\infty} Y_{n}(t), t \in \mathbb{R}$; then

$$
Y(t)=\left(\Lambda_{2} f\right)(t)=\int_{-\infty}^{t} S(t-s) f_{2}(s) d s, \quad t \in \mathbb{R}
$$

It is obvious that $Y(t) \in B C(\mathbb{R}, X)$. So, we need to show that

$$
\lim _{T \rightarrow \infty} \frac{1}{\mu\left(T, \rho_{1}\right)} \int_{-T}^{T} \rho_{2}(t)\|Y(t)\| d t=0
$$


In fact, by Hölder inequality,

$$
\begin{aligned}
\left\|Y_{n}(t)\right\| & \leq \int_{n-1}^{n} \phi(s)\left\|f_{2}(t-s)\right\| d s \\
& \leq \phi(n-1) \int_{t-n}^{t-n+1}\left\|f_{2}(s)\right\| d s \\
& \leq \phi(0)\left(\int_{t-n}^{t-n+1}\left\|f_{2}(s)\right\|^{p} d s\right)^{1 / p},
\end{aligned}
$$

then

$$
\begin{aligned}
& \frac{1}{\mu\left(T, \rho_{1}\right)} \int_{-T}^{T} \rho_{2}(t)\left\|Y_{n}(t)\right\| d t \\
& \quad \leq \frac{\phi(0)}{\mu\left(T, \rho_{1}\right)} \int_{-T}^{T} \rho_{2}(t)\left(\int_{t-n}^{t-n+1}\left\|f_{2}(s)\right\|^{p} d s\right)^{1 / p} d t
\end{aligned}
$$

hence $Y_{n} \in W P P_{0}\left(\mathbb{R}, X, \rho_{1}, \rho_{2}\right)$. By Lemma 24 , it follows that $Y(t) \in W P P_{0}\left(\mathbb{R}, X, \rho_{1}, \rho_{2}\right)$.

By $f_{1}(t+\omega)-f_{1}(t)=0$ a.e. $t \in \mathbb{R}$, one has

$$
\left(\Lambda_{1} f\right)(t+\omega)=\int_{-\infty}^{t+\omega} S(t+\omega-s) f_{1}(s) d s=\left(\Lambda_{1} f\right)(t)
$$

Hence $\Lambda f \in W P P_{\omega}\left(\mathbb{R}, X, \rho_{1}, \rho_{2}\right)$. The proof is complete.

\section{Fractional Integrodifferential Equation}

This section is devoted to the existence and uniqueness of weighted pseudoperiodic solutions of the following fractional integrodifferential equation:

$$
\begin{gathered}
D_{t}^{\alpha} u(t)=A u(t)+D_{t}^{\alpha-1} f(t, u(t), K u(t)), \\
t \in \mathbb{R}, 1<\alpha<2, \\
K u(t)=\int_{-\infty}^{t} k(t-s) g(s, u(s)) d s,
\end{gathered}
$$

where $A: D(A) \subset X \rightarrow X$ is a linear densely operator of sectorial type on a complex Banach space $(X,\|\cdot\|)$ and $f, g$, and $k$ are suitable functions. The fractional derivative $D_{t}^{\alpha}$ is to be understood in the Riemann-Liouville sense.

Before starting our main results, we recall the definition of the mild solution to (62).

Definition 37 (see $[8,30]$ ). Assume that $A$ generates an integral solution operator $S_{\alpha}(t)$. A continuous function $u$ : $\mathbb{R} \rightarrow X$ satisfying the integral equation

$$
u(t)=\int_{-\infty}^{t} s_{\alpha}(t-s) f(s, u(s), K u(s)) d s, \quad t \in \mathbb{R}
$$

is called a mild solution on $\mathbb{R}$ to $(62)$.

To study (62), we introduce the following assumptions.
$\left(H_{1}\right) A$ is a sectorial operator of type $\widetilde{\omega}<0$ with $0<\theta<$ $\pi(1-\alpha / 2)$.

$\left(H_{2}\right) k(t) \in L^{1}\left(\mathbb{R}^{+}\right)$is a continuous, nonincreasing function.

$\left(H_{3}\right) g=g_{1}+g_{2} \in S^{p} W P P_{\omega}\left(\mathbb{R} \times X, X, \rho_{1}, \rho_{2}\right)$ with $g_{2} \in$ $S^{p} W P P_{0}\left(\mathbb{R} \times X, X, \rho_{1}, \rho_{2}\right), g_{1}(t+\omega, u)-g_{1}(t, u)=0$ a.e. $t \in \mathbb{R}, u \in X$, and there exist constants $L_{g}, L_{g_{1}}>0$ such that

$$
\|g(t, u)-g(t, v)\| \leq L_{g}\|u-v\|,
$$

$$
\left\|g_{1}(t, u)-g_{1}(t, v)\right\| \leq L_{g_{1}}\|u-v\|
$$

$$
t \in \mathbb{R}, u, v \in X
$$

$\left(H_{4}\right) f=f_{1}+f_{2} \in S^{p} W P P_{\omega}\left(\mathbb{R} \times X \times X, X, \rho_{1}, \rho_{2}\right)$ with $f_{2} \in S^{p} W P P_{0}\left(\mathbb{R} \times X \times X, X, \rho_{1}, \rho_{2}\right), f_{1}(t+\omega, u, v)-$ $f_{1}(t, u, v)=0$ a.e. $t \in \mathbb{R},(u, v) \in X \times X$, and there exist constants $L_{f}, L_{f_{1}}>0$ such that

$$
\begin{aligned}
& \left\|f\left(t, u_{1}, u_{2}\right)-f\left(t, v_{1}, v_{2}\right)\right\| \\
& \quad \leq L_{f}\left(\left\|u_{1}-v_{1}\right\|+\left\|u_{2}-v_{2}\right\|\right),
\end{aligned}
$$

$$
t \in \mathbb{R}, u_{1}, u_{2}, v_{1}, v_{2} \in X
$$

$$
\begin{array}{r}
\left\|f_{1}\left(t, u_{1}, u_{2}\right)-f_{1}\left(t, v_{1}, v_{2}\right)\right\| \\
\leq L_{f_{1}}\left(\left\|u_{1}-v_{1}\right\|+\left\|u_{2}-v_{2}\right\|\right), \\
t \in \mathbb{R}, u_{1}, u_{2}, v_{1}, v_{2} \in X .
\end{array}
$$

$\left(H_{5}\right) \rho_{1}, \rho_{2} \in U_{T}, \inf _{T>0}\left(\mu\left(T, \rho_{2}\right) / \mu\left(T, \rho_{1}\right)\right)=\delta_{0}>0$ and $\sup _{T>0}\left(\mu\left(T, \rho_{2}\right) / \mu\left(T, \rho_{1}\right)\right)<\infty$.

Lemma 38. If $u \in W P P_{\omega}\left(\mathbb{R}, X, \rho_{1}, \rho_{2}\right)$, assuming that $\left(H_{1}\right)$, $\left(H_{2}\right),\left(H_{3}\right)$, and $\left(H_{5}\right)$ hold, then $K u \in W P P_{\omega}\left(\mathbb{R}, X, \rho_{1}, \rho_{2}\right)$.

Proof. Since $g \in S^{p} W P P_{\omega}\left(\mathbb{R}, X, \rho_{1}, \rho_{2}\right)$, by $\left(H_{3}\right)$ and Theorem 34, it is clear that $\psi(\cdot):=g(\cdot, u(\cdot)) \in S^{p} W P P_{\omega}(\mathbb{R} \times$ $\left.X, X, \rho_{1}, \rho_{2}\right)$. Similarly as the proof of Lemma $36, K u \in$ $W P P_{\omega}\left(\mathbb{R}, X, \rho_{1}, \rho_{2}\right)$.

Lemma 39. Assume that $h \in S^{p} W P P_{\omega}\left(\mathbb{R}, X, \rho_{1}, \rho_{2}\right)$ and $\left(H_{5}\right)$ holds; then

$$
(\Lambda h)(t):=\int_{-\infty}^{t} S_{\alpha}(t-s) h(s) d s, \quad t \in \mathbb{R}
$$

lies in $W P P_{\omega}\left(\mathbb{R}, X, \rho_{1}, \rho_{2}\right)$.

Proof. By (4), one has

$$
\left\|S_{\alpha}(t)\right\| \leq \frac{C M}{1+|\widetilde{\omega}| t^{\alpha}}, \quad t \geq 0 .
$$

Since $1<\alpha<2$ and $C M /\left(1+|\widetilde{\omega}| t^{\alpha}\right) \in L^{1}\left(\mathbb{R}^{+}\right)$is nonincreasing, and by Lemma $36, \Lambda h \in W P P_{\omega}\left(\mathbb{R}, X, \rho_{1}, \rho_{2}\right)$. 
Theorem 40. Assume that $\left(H_{1}\right)-\left(H_{5}\right)$ hold; if

$$
C M L_{f}\left(1+L_{g}\|k\|_{L^{1}}\right)|\widetilde{\omega}|^{-1 / \alpha} \pi<\alpha \sin \left(\frac{\pi}{\alpha}\right),
$$

then (62) has a unique $W P P_{\omega}$ mild solution.

Proof. Define the operator $\mathbb{F}: W P P_{\omega}\left(\mathbb{R}, X, \rho_{1}, \rho_{2}\right) \rightarrow W P P_{\omega}$ $\left(\mathbb{R}, X, \rho_{1}, \rho_{2}\right)$ by

$$
(\mathbb{F} u)(t)=\int_{-\infty}^{t} S_{\alpha}(t-s) f(s, u(s), K u(s)) d s, \quad t \in \mathbb{R} .
$$

First, we show that $\mathbb{F}$ is well defined. In fact, if $u \in W P P_{\omega}\left(\mathbb{R}, X, \rho_{1}, \rho_{2}\right)$, by Lemma 38,Ku $\in W P P_{\omega}$ $\left(\mathbb{R}, X, \rho_{1}, \rho_{2}\right) \subset S^{p} W P P_{\omega}\left(\mathbb{R}, X, \rho_{1}, \rho_{2}\right)$, so $h(\cdot):=f(\cdot$, $u(\cdot), K u(\cdot)) \in S^{p} W P P_{\omega}\left(\mathbb{R}, X, \rho_{1}, \rho_{2}\right)$ from Theorem 34. By Lemma 39, it is not difficult to see that $\mathbb{F}$ is well defined.

For any $u, v \in W P P_{\omega}\left(\mathbb{R}, X, \rho_{1}, \rho_{2}\right)$,

$$
\begin{aligned}
& \|(\mathbb{F} u)(t)-(\mathbb{F} v)(t)\| \\
& \leq \int_{-\infty}^{t}\left\|S_{\alpha}(t-s)\right\| \\
& \quad \times\|f(s, u(s), K u(s))-f(s, v(s), K v(s))\| d s \\
& \leq L_{f} \int_{-\infty}^{t}\left\|S_{\alpha}(t-s)\right\| \\
& \quad \times(\|u(s)-v(s)\|+\|K u(s)-K v(s)\|) d s \\
& \leq L_{f}\left(1+L_{g}\|k\|_{L^{1}}\right) \int_{-\infty}^{t}\left\|S_{\alpha}(t-s)\right\|\|u(s)-v(s)\| d s \\
& \leq L_{f}\left(1+L_{g}\|k\|_{L^{1}}\right)\|u-v\| \int_{0}^{\infty} \frac{C M}{1+|\widetilde{\omega}| s^{\alpha}} d s \\
& \leq \frac{C M L_{f}\left(1+L_{g}\|k\|_{L^{1}}\right)|\widetilde{\omega}|^{-1 / \alpha} \pi}{\alpha \sin (\pi / \alpha)}\|u-v\| .
\end{aligned}
$$

By the Banach contraction mapping principle, $\mathbb{F}$ has a unique fixed point in $W P P_{\omega}\left(\mathbb{R}, X, \rho_{1}, \rho_{2}\right)$, which is the unique $W P P_{\omega}$ mild solution to $(62)$.

Remark 41. It is not difficult to see that similar result of Theorem 40 holds for $W P P_{\text {wap }}$ mild solution. In this case, $S^{p} W P P_{\omega a p}\left(\mathbb{R}, X, \rho_{1}, \rho_{2}\right)$ instead of $S^{p} W P P_{\omega}\left(\mathbb{R}, X, \rho_{1}, \rho_{2}\right)$ in $\left(H_{3}\right),\left(H_{4}\right)$, then $(62)$ has a unique $W P P_{\text {wap }}$ mild solution.

\section{Nonautonomous Differential Equation}

This section is devoted to the existence and uniqueness of weighted pseudoperiodic solutions of the following nonautonomous differential equation in Banach space $X$ :

$$
u^{\prime}(t)=A(t) u(t)+f(t, u(t)), \quad t \in \mathbb{R},
$$

where $A(t)$ satisfies "Acquistapace-Terreni" conditions, evolution family $(U(t, s))_{t \geq s}$ generated by $A(t)$ has exponential dichotomy, and $f$ is a suitable function.

In this section, we consider the following assumptions.
$\left(A_{1}\right)$ There exists constants $\lambda_{0} \geq 0, \theta \in(\pi / 2, \pi), L, \widetilde{M} \geq 0$ and $\beta, \gamma \in(0,1)$ with $\beta+\gamma>1$ such that

$$
\begin{gathered}
\Sigma_{\theta} \cup\{0\} \subset \rho\left(A(t)-\lambda_{0}\right), \\
\left\|R\left(\lambda, A(t)-\lambda_{0}\right)\right\| \leq \frac{\widetilde{M}}{1+|\lambda|}, \\
\left\|\left(A(t)-\lambda_{0}\right) R\left(\lambda, A(t)-\lambda_{0}\right)\left[R\left(\lambda_{0}, A(t)\right)-R\left(\lambda_{0}, A(s)\right)\right]\right\| \\
\leq L|t-s|^{\beta}|\lambda|^{-\gamma}
\end{gathered}
$$

for $t, s \in \mathbb{R}, \Sigma_{\theta}=\{\lambda \in \mathbb{C} \backslash\{0\}:|\arg \lambda| \leq \theta\}$.

$\left(A_{2}\right)$ The evolution family $(U(t, s))_{t \geq s}$ generated by $A(t)$ has an exponential dichotomy with constants $c>0$ and $\delta>0$, dichotomy projections $P(t), t \in \mathbb{R}$, and Green's function $\Gamma(t, s)$.

$\left(A_{3}\right)$ There exists $\omega \in \mathbb{R} \backslash\{0\}$ such that $\Gamma(t+\omega, s+\omega)=$ $\Gamma(t, s)$.

$\left(A_{4}\right) \quad f=f_{1}+f_{2} \in S^{p} W P P_{\omega}\left(\mathbb{R} \times X, X, \rho_{1}, \rho_{2}\right)$ with $f_{2} \in$ $S^{p} W P P_{0}\left(\mathbb{R} \times X, X, \rho_{1}, \rho_{2}\right), f_{1}(t+\omega, u)-f_{1}(t, u)=0$ a.e. $t \in \mathbb{R}, u \in X$, and there exist constants $L_{f}, L_{f_{1}}>0$ such that

$$
\begin{array}{r}
\|f(t, u)-f(t, v)\| \leq L_{f}\|u-v\|, \\
\left\|f_{1}(t, u)-f_{1}(t, v)\right\| \leq L_{f_{1}}\|u-v\|, \\
t \in \mathbb{R}, u, v \in X .
\end{array}
$$

$\left(A_{5}\right) \rho_{1}, \rho_{2} \in U_{T}, \inf _{T>0}\left(\mu\left(T, \rho_{2}\right) / \mu\left(T, \rho_{1}\right)\right)=\delta_{0}>0$ and $\sup _{T>0}\left(\mu\left(T, \rho_{2}\right) / \mu\left(T, \rho_{1}\right)\right)<\infty$.

Remark 42. $\left(A_{1}\right)$ is usually called "Acquistapace-Terreni" conditions, which was first introduced in [31] and widely used to study nonautonomous differential equations in [19$21,31,32]$. If $\left(A_{1}\right)$ holds, there exists a unique evolution family $(U(t, s))_{t \geq s}$ on $X$, which governs the homogeneous version of (71) [32].

Definition 43 (see [21]). A mild solution of (71) is a continuous function $u: \mathbb{R} \rightarrow X$ satisfying

$$
u(t)=U(t, s) u(s)+\int_{s}^{t} U(t, \sigma) f(\sigma, u(\sigma)) d \sigma
$$

for all $t \geq s, t, s \in \mathbb{R}$.

Lemma 44. Assume that $f \in S^{p} W P P_{\omega}\left(\mathbb{R}, X, \rho_{1}, \rho_{2}\right)$ and $\left(A_{5}\right)$ hold; then

$$
\begin{aligned}
(\Pi f)(t)= & \int_{-\infty}^{t} U(t, \sigma) P(\sigma) f(\sigma) d \sigma \\
& -\int_{t}^{\infty} U_{Q}(t, \sigma) Q(\sigma) f(\sigma) d \sigma
\end{aligned}
$$

lies in $W P P_{\omega}\left(\mathbb{R}, X, \rho_{1}, \rho_{2}\right)$. 
Abstract and Applied Analysis

11

Proof. Let $f(t)=f_{1}(t)+f_{2}(t)$, where $f_{2} \in S^{p} W P P_{0}(\mathbb{R}$, $\left.X, \rho_{1}, \rho_{2}\right)$ and $f_{1}(t+\omega)-f_{1}(t)=0$ ae. $t \in \mathbb{R}$; then

$$
\begin{aligned}
(\Pi f)(t)= & \int_{-\infty}^{t} U(t, \sigma) P(\sigma) f(\sigma) d \sigma \\
& -\int_{t}^{\infty} U_{Q}(t, \sigma) Q(\sigma) f(\sigma) d \sigma \\
:= & \left(\Pi_{1} f\right)(t)+\left(\Pi_{2} f\right)(t),
\end{aligned}
$$

where

$$
\begin{aligned}
\left(\Pi_{1} f\right)(t)= & \int_{-\infty}^{t} U(t, \sigma) P(\sigma) f_{1}(\sigma) d \sigma \\
& -\int_{t}^{\infty} U_{Q}(t, \sigma) Q(\sigma) f_{1}(\sigma) d \sigma, \\
\left(\Pi_{2} f\right)(t)= & \int_{-\infty}^{t} U(t, \sigma) P(\sigma) f_{2}(\sigma) d \sigma \\
& \quad-\int_{t}^{\infty} U_{Q}(t, \sigma) Q(\sigma) f_{2}(\sigma) d \sigma .
\end{aligned}
$$

Next, we show that $\Pi_{2} f \in W P P_{0}\left(\mathbb{R}, X, \rho_{1}, \rho_{2}\right)$. Consider the integrals

$$
\begin{aligned}
X_{n}(t)= & \int_{t-n}^{t-n+1} U(t, \sigma) P(\sigma) f_{2}(\sigma) d \sigma \\
& -\int_{t+n-1}^{t+n} U_{Q}(t, \sigma) Q(\sigma) f_{2}(\sigma) d \sigma \\
= & \int_{n-1}^{n} U(t, t-\sigma) P(t-\sigma) f_{2}(t-\sigma) d \sigma \\
& -\int_{n-1}^{n} U_{Q}(t, t+\sigma) Q(t+\sigma) f_{2}(t+\sigma) d \sigma
\end{aligned}
$$

for each $t \in \mathbb{R}, n=1,2, \ldots$.

By the Hölder inequality and the exponential dichotomy of $(U(t, s))_{t \geq s}$, it follows that

$$
\begin{aligned}
\left\|X_{n}(t)\right\| \leq & c \int_{t-n}^{t-n+1} e^{-\delta(t-\sigma)}\left\|f_{2}(\sigma)\right\| d \sigma \\
& +c \int_{t+n-1}^{t+n} e^{-\delta(\sigma-t)}\left\|f_{2}(\sigma)\right\| d \sigma \\
\leq & c\left(\int_{t-n}^{t-n+1} e^{-q \delta(t-\sigma)} d \sigma\right)^{1 / q}\left(\int_{t-n}^{t-n+1}\left\|f_{2}(\sigma)\right\|^{p} d \sigma\right)^{1 / p} \\
& +c\left(\int_{t+n-1}^{t+n} e^{-q \delta(\sigma-t)} d \sigma\right)^{1 / q}\left(\int_{t+n-1}^{t+n}\left\|f_{2}(\sigma)\right\|^{p} d \sigma\right)^{1 / p} \\
\leq & \frac{c}{\sqrt[q]{q \delta}}\left(e^{-q \delta(n-1)}-e^{-q \delta n}\right)^{1 / q}\left\|f_{2}\right\|_{S^{p}} \\
& +\frac{c}{\sqrt[q]{q \delta}}\left(e^{-q \delta(n-1)}-e^{-q \delta n}\right)^{1 / q_{1}}\left\|f_{2}\right\|_{S^{p}} \\
\leq & 2 c e^{-\delta n} \sqrt[\frac{e^{q \delta}+1}{q \delta}]{\frac{q \delta}{f_{2}} \|_{S^{p}},}
\end{aligned}
$$

where $q=p /(p-1)$. Since the series $2 c \sqrt[q]{\left(e^{q \delta}+1\right) /(q \delta)} \sum_{n=1}^{\infty} e^{-\delta n}$ is convergent, by the Weierstrass test, $\sum_{n=1}^{\infty} X_{n}(t)$ is uniformly convergent on $\mathbb{R}$. Furthermore,

$$
\begin{aligned}
X(t)=\left(\Pi_{2} f\right)(t)= & \int_{-\infty}^{t} U(t, \sigma) P(\sigma) f_{2}(\sigma) d \sigma \\
& -\int_{t}^{\infty} U_{\mathrm{Q}}(t, \sigma) Q(\sigma) f_{2}(\sigma) d \sigma .
\end{aligned}
$$

It is obvious that $X(t) \in B C(\mathbb{R}, X)$. So, we need to show that

$$
\lim _{T \rightarrow \infty} \frac{1}{\mu\left(T, \rho_{1}\right)} \int_{-T}^{T} \rho_{2}(t)\|X(t)\| d t=0 .
$$

In fact, by Hölder inequality, one has

$$
\begin{aligned}
& \left\|X_{n}(t)\right\| \leq \int_{n-1}^{n}\left\|U(t, t-\sigma) P(t-\sigma) f_{2}(t-\sigma)\right\| d \sigma \\
& +\int_{n-1}^{n}\left\|U_{Q}(t, t+\sigma) Q(t+\sigma) f_{2}(t+\sigma)\right\| d \sigma \\
& \leq c \int_{n-1}^{n} e^{-\delta \sigma}\left\|f_{2}(t-\sigma)\right\| d \sigma \\
& +c \int_{n-1}^{n} e^{-\delta \sigma}\left\|f_{2}(t+\sigma)\right\| d \sigma \\
& \leq c\left(\int_{n-1}^{n} e^{-q \delta \sigma} d \sigma\right)^{1 / q}\left(\int_{n-1}^{n}\left\|f_{2}(t-\sigma)\right\|^{p} d \sigma\right)^{1 / p} \\
& +c\left(\int_{n-1}^{n} e^{-q \delta \sigma} d \sigma\right)^{1 / q}\left(\int_{n-1}^{n}\left\|f_{2}(t+\sigma)\right\|^{p} d \sigma\right)^{1 / p} \\
& \leq c e \sqrt[-\delta n]{\frac{q}{q \delta}+1}\left(\int_{t-n}^{t-n+1}\left\|f_{2}(\sigma)\right\|^{p} d \sigma\right)^{1 / p} \\
& +c e \sqrt[-\delta n]{\frac{q}{e^{q \delta}+1}}\left(\int_{t+n-1}^{t+n}\left\|f_{2}(\sigma)\right\|^{p} d \sigma\right)^{2 / p} \\
& \leq C_{q}(c, \delta)\left(\int_{t-n}^{t-n+1}\left\|f_{2}(\sigma)\right\|^{p} d \sigma\right)^{1 / p} \\
& +C_{q}(c, \delta)\left(\int_{t+n-1}^{t+n}\left\|f_{2}(\sigma)\right\|^{p} d \sigma\right)^{1 / p},
\end{aligned}
$$

where $C_{q}(c, \delta)$ is a constant, depending on $q, c$, and $\delta$. Then

$$
\begin{aligned}
& \frac{1}{\mu\left(T, \rho_{1}\right)} \int_{-T}^{T} \rho_{2}(t)\left\|X_{n}(t)\right\| d t \\
& \leq \frac{C_{q}(c, \delta)}{\mu\left(T, \rho_{1}\right)} \int_{-T}^{T} \rho_{2}(t)\left(\int_{t-n}^{t-n+1}\left\|f_{2}(\sigma)\right\|^{p} d \sigma\right)^{1 / p} d t \\
& \quad+\frac{C_{q}(c, \delta)}{\mu\left(T, \rho_{1}\right)} \int_{-T}^{T} \rho_{2}(t)\left(\int_{t+n-1}^{t+n}\left\|f_{2}(\sigma)\right\|^{p} d \sigma\right)^{1 / p} d t
\end{aligned}
$$


hence $X_{n} \in W P P_{0}\left(\mathbb{R}, X, \rho_{1}, \rho_{2}\right)$. By Lemma 24 , it follows that $\Pi_{2} f \in W P P_{0}\left(\mathbb{R}, X, \rho_{1}, \rho_{2}\right)$. It is not difficult to see that $\left(\Pi_{1} f\right)(t+\omega)-\left(\Pi_{1} f\right)(t)=0$ a.e. $t \in \mathbb{R}$, whence $\Pi f \in$ $W P P_{\omega}\left(\mathbb{R}, X, \rho_{1}, \rho_{2}\right)$.

Theorem 45. Assume that $\left(A_{1}\right)-\left(A_{5}\right)$ hold; if $2 c L_{f}<\delta$, then (71) has a unique mild solution $u \in W P P_{\omega}\left(\mathbb{R}, X, \rho_{1}, \rho_{2}\right)$ such that

$$
\begin{aligned}
u(t)= & \int_{-\infty}^{t} U(t, \sigma) P(\sigma) f(\sigma, u(\sigma)) d \sigma \\
& -\int_{t}^{\infty} U_{\mathrm{Q}}(t, \sigma) Q(\sigma) f(\sigma, u(\sigma)) d \sigma, \quad t \in \mathbb{R} .
\end{aligned}
$$

Proof. First, we show that (71) admits a unique bounded solution given by (84), which is similar to the proof of [21, Theorem 3.3]. For $u \in W P P_{\omega}\left(\mathbb{R}, X, \rho_{1}, \rho_{2}\right)$, it is clear that $h(\cdot):=f(\cdot, u(\cdot)) \in S^{p} W P P_{\omega}\left(\mathbb{R}, X, \rho_{1}, \rho_{2}\right)$ since $\left(A_{4}\right)$ holds; then by the definition of exponential dichotomy of $(U(t, s))_{t \geq s}$, it is not difficult to see that (84) is well defined for each $t \in \mathbb{R}$.

For all $t \geq s, t, s \in \mathbb{R}$,

$$
\begin{aligned}
u(s)= & \int_{-\infty}^{s} U(s, \sigma) P(\sigma) h(\sigma) d \sigma \\
& -\int_{s}^{\infty} U_{Q}(s, \sigma) Q(\sigma) h(\sigma) d \sigma, \quad s \in \mathbb{R} ;
\end{aligned}
$$

then

$$
\begin{aligned}
U(t, s) u(s)= & \int_{-\infty}^{s} U(t, s) U(s, \sigma) P(\sigma) h(\sigma) d \sigma \\
& -\int_{s}^{\infty} U(t, s) U_{\mathrm{Q}}(s, \sigma) Q(\sigma) h(\sigma) d \sigma \\
= & \int_{-\infty}^{s} U(t, \sigma) P(\sigma) h(\sigma) d \sigma \\
& -\int_{s}^{\infty} U_{\mathrm{Q}}(t, \sigma) Q(\sigma) h(\sigma) d \sigma \\
= & \int_{-\infty}^{t} U(t, \sigma) P(\sigma) h(\sigma) d \sigma \\
& -\int_{s}^{t} U(t, \sigma) P(\sigma) h(\sigma) d \sigma \\
& -\int_{t}^{\infty} U_{\mathrm{Q}}(t, \sigma) Q(\sigma) h(\sigma) d \sigma \\
& -\int_{s}^{t} U_{\mathrm{Q}}(t, \sigma) Q(\sigma) h(\sigma) d \sigma \\
= & u(t)-\int_{s}^{t} U(t, \sigma) h(\sigma) d \sigma
\end{aligned}
$$

so $u$ is a mild solution of (71). To prove the uniqueness, let $v$ be another mild solution of (71), then

$$
v(t)=U(t, s) u(s)+\int_{s}^{t} U(t, \sigma) h(\sigma) d \sigma,
$$

by the exponential dichotomy of $(U(t, s))_{t \geq s}$,

$$
P(t) v(t)=\int_{-\infty}^{t} U(t, \sigma) P(\sigma) h(\sigma) d \sigma, \quad t \in \mathbb{R} .
$$

Similarly,

$$
Q(t) v(t)=\int_{\infty}^{t} U_{\mathrm{Q}}(t, \sigma) Q(\sigma) h(\sigma) d \sigma, \quad t \in \mathbb{R} .
$$

So

$$
\begin{aligned}
v(t)= & P(t) v(t)+Q(t) v(t) \\
= & \int_{-\infty}^{t} U(t, \sigma) P(\sigma) h(\sigma) d \sigma \\
& -\int_{t}^{\infty} U_{Q}(t, \sigma) Q(\sigma) h(\sigma) d \sigma \\
= & u(t) .
\end{aligned}
$$

Next, define the operator $\mathscr{F}: W P P_{\omega}\left(\mathbb{R}, X, \rho_{1}, \rho_{2}\right) \rightarrow$ $W P P_{\omega}\left(\mathbb{R}, X, \rho_{1}, \rho_{2}\right)$ by

$$
\begin{aligned}
(\mathscr{F} u)(t)= & \int_{-\infty}^{t} U(t, \sigma) P(\sigma) f(\sigma, u(\sigma)) d \sigma \\
& -\int_{t}^{\infty} U_{\mathrm{Q}}(t, \sigma) Q(\sigma) f(\sigma, u(\sigma)) d \sigma, \quad t \in \mathbb{R} .
\end{aligned}
$$

If $u \in W P P_{\omega}\left(\mathbb{R}, X, \rho_{1}, \rho_{2}\right)$, it is not difficult to see that $f(\cdot, u(\cdot))$ $\in S^{p} W P P_{\omega}\left(\mathbb{R}, X, \rho_{1}, \rho_{2}\right)$ since $\left(A_{4}\right)$ holds. By Lemma 44 , $\mathscr{F} u \in W P P_{\omega}\left(\mathbb{R}, X, \rho_{1}, \rho_{2}\right)$, so $\mathscr{F}$ is well defined.

For any $u, v \in W P P_{\omega}\left(\mathbb{R}, X, \rho_{1}, \rho_{2}\right)$,

$$
\begin{aligned}
& \|(\mathscr{F} u)(t)-(\mathscr{F} v)(t)\| \\
& \leq \int_{-\infty}^{t}\|U(t, \sigma) P(\sigma)[f(\sigma, u(\sigma))-f(\sigma, v(\sigma))]\| d \sigma \\
& \quad+\int_{t}^{\infty}\left\|U_{Q}(t, \sigma) Q(\sigma)[f(\sigma, u(\sigma))-f(\sigma, v(\sigma))]\right\| d \sigma \\
& \leq L_{f} \int_{-\infty}^{t} c e^{-\delta(t-\sigma)}\|u(\sigma)-v(\sigma)\| d \sigma \\
& \quad+L_{f} \int_{t}^{\infty} c e^{-\delta(\sigma-t)}\|u(\sigma)-v(\sigma)\| d \sigma \\
& \leq \frac{2 c}{\delta} L_{f}\|u-v\| .
\end{aligned}
$$

By the Banach contraction mapping principle, $\mathscr{F}$ has a unique fixed point in $W P P_{\omega}\left(\mathbb{R}, X, \rho_{1}, \rho_{2}\right)$, which is the unique $W P P_{\omega}$ mild solution to (71).

Remark 46. It is not difficult to see that similar result of Theorem 45 holds for $W P P_{\text {wap }}$ mild solution. In this case, $S^{p} W P P_{\omega a p}\left(\mathbb{R}, X, \rho_{1}, \rho_{2}\right)$ instead of $S^{p} W P P_{\omega}\left(\mathbb{R}, X, \rho_{1}, \rho_{2}\right)$ in $\left(A_{4}\right)$; then (71) has a unique $W P P_{\text {wap }}$ mild solution. 


\section{Example}

In this section, we provide some examples to illustrate our main results.

Example 1. Consider the fractional partial differential equation

$$
\begin{array}{rl}
D_{t}^{\alpha} w(t, x)= & \frac{\partial^{2} w(t, x)}{\partial x^{2}}-a w(t, x) \\
& +D_{t}^{\alpha-1} f(t, x, w(t, x), K w(t, x)), \\
t & t \mathbb{R}, x \in(0,1), \\
K w(t, x)= & \int_{-\infty}^{t} k(t-s) g(s, x, w(s, x)) d s, \\
& w(t, 0)=w(t, 1)=0,
\end{array}
$$

where $1<\alpha<2, a>0$ and $k(\cdot)$ is a real valued continuous nonincreasing function such that $|k(t)| \leq C_{k} e^{-b t}$ for $t \geq 0$ and $C_{k}$ and $b$ are positive constants.

Take $X=L^{2}[0,1]$, and define the operator $A$ by

$$
\begin{gathered}
D(A):=\left\{w \in L^{2}[0,1]: w^{\prime \prime} \in L^{2}[0,1], w(0)=w(1)=0\right\} \\
A w=w^{\prime \prime}-a w, \quad \forall w \in D(A) .
\end{gathered}
$$

It is well know that $A$ is the infinitesimal generator of an analytic semigroup on $L^{2}[0,1]$. Thus $A$ is of sectorial type $\widetilde{\omega}=-a<0$.

Let $u(t)=w(t, x), t \in \mathbb{R}, x \in[0,1]$; then (93) can be rewritten as the abstract form (62). Take $\rho_{1}(t)=e^{t}$ and $\rho_{2}(t)=$ $1+t^{2}$; by Theorem 40 , one has the following.

Theorem 47. Under assumptions $\left(H_{3}\right)$ and $\left(H_{4}\right)$, then (93) has a unique $W P P_{\omega}\left(\mathbb{R}, X, \rho_{1}, \rho_{2}\right)$ mild solution whenever

$$
C L_{f}\left(1+L_{g}\|k\|_{L^{1}}\right)|a|^{-1 / \alpha} \pi<\alpha \sin \left(\frac{\pi}{\alpha}\right)
$$

Example 2. Consider the heat equation with Dirichlet conditions

$$
\begin{gathered}
\frac{\partial u(t, x)}{\partial t}=\frac{\partial^{2} u(t, x)}{\partial x^{2}}+q(t, x) u(t, x)+f(t, u(t, x)), \\
t \in \mathbb{R}, x \in[0, \pi], \\
u(t, 0)=u(t, \pi)=0, \quad t \in \mathbb{R},
\end{gathered}
$$

where $f: \mathbb{R} \times L^{2}[0, \pi] \rightarrow L^{2}[0, \pi]$ is weighted pseudoperiodic function, $q: \mathbb{R} \times[0, \pi] \rightarrow \mathbb{R}$ is a continuous function, $q(t+\omega, x)=q(t, x)$ for $\omega>0$, and there exists $\gamma_{0}>0$ such that $q(t, x) \leq-\gamma_{0}$.
Take $X=L^{2}[0, \pi]$ being equipped with its natural topology and define

$$
\begin{gathered}
D(A)=\left\{u \in L^{2}[0, \pi]: u^{\prime \prime} \in L^{2}[0, \pi], u(0)=u(\pi)=0\right\}, \\
A u=u^{\prime \prime}, \quad \forall u \in D(A) .
\end{gathered}
$$

Let $\varphi_{n}(t)=\sqrt{2 / \pi} \sin (n t)$ for all $n \in \mathbb{R}$. It is well known that $A$ is the infinitesimal generator of an analytic semigroup $(T(t))_{t \geq 0}$ on $L^{2}[0, \pi]$ with $\|T(t)\| \leq e^{-t}$ for $t \geq 0$. Moreover,

$$
T(t) \varphi=\sum_{n=1}^{\infty} e^{-n^{2} t}\left\langle\varphi, \varphi_{n}\right\rangle \varphi_{n}
$$

for each $\varphi \in L^{2}[0, \pi]$.

Define a family of linear operators $A(t)$ by

$$
\begin{gathered}
D(A(t))=D(A), \\
A(t) \varphi(x)=A \varphi(x)+q(t, x) \varphi, \quad \forall x \in[0, \pi], \varphi \in D(A) .
\end{gathered}
$$

Since $q(t, x) \leq-\gamma_{0}$, one can see that the system

$$
\begin{gathered}
u^{\prime}(t)=A(t) u(t), \quad t \geq s, \\
u(s)=\varphi \in L^{2}[0, \pi]
\end{gathered}
$$

has an associated evolution family $(U(t, s))_{t \geq s}$ on $L^{2}[0, \pi]$, which can be explicitly expressed by

$$
U(t, s) \varphi=T(t-s) e^{\int_{s}^{t} q(\tau, x) d \tau} \varphi .
$$

Moreover,

$$
\|U(t, s)\| \leq e^{-\left(1+\gamma_{0}\right)(t-s)} \quad \text { for every } t \geq s .
$$

It is not difficult to verify that $A(t)$ satisfy $\left(A_{1}\right)-\left(A_{3}\right)$ with $c=$ $1, \delta=1+\gamma_{0}$. One can see $[21,33]$ for more details.

By Theorem 45, we claim that

Theorem 48. Under the assumptions $\left(A_{4}\right)$ and $\left(A_{5}\right)$, (96) admits a unique $W P P_{\omega}$ mild solution $u(t) \in W P P_{\omega}$ $\left(\mathbb{R}, L^{2}[0, \pi], \rho_{1}, \rho_{2}\right)$ if $2 L_{f}<1+\gamma_{0}$.

\section{Conflict of Interests}

The author declares that there is no conflict of interests regarding the publication of this paper.

\section{Acknowledgment}

This material is based upon work funded by Zhejiang Provincial Natural Science Foundation of China under Grant no. LQ13A010015. 


\section{References}

[1] N. S. Al-Islam, S. M. Alsulami, and T. Diagana, "Existence of weighted pseudo anti-periodic solutions to some non-autonomous differential equations," Applied Mathematics and Computation, vol. 218, no. 11, pp. 6536-6548, 2012.

[2] W. Stepanov, "Sur quelques généralizations des fonctions presque-périodiques," Comptes Rendus de l'Académie des Sciences Paris, vol. 181, pp. 90-94, 1925.

[3] W. Stepanov, "Über einige verallgemeinerungen der fastperiodischen funktionen," Mathematische Annalen, vol. 90, pp. 473492, 1925.

[4] V. Casarino, "Almost automorphic groups and semigroups," Rendiconti della Accademia Nazionale delle Scienze detta dei XL. Memorie di Matemàtica, vol. 24, pp. 219-235, 2000.

[5] T. Diagana, "Stepanov-like pseudo almost periodic functions and their applications to differential equations," Communications in Mathematical Analysis, vol. 3, no. 1, pp. 9-18, 2007.

[6] Z. Xia and M. Fan, "Weighted Stepanov-like pseudo almost automorphy and applications," Nonlinear Analysis. Theory, Methods \& Applications A, vol. 75, no. 4, pp. 2378-2397, 2012.

[7] S. Abbas, "Pseudo almost automorphic solutions of some nonlinear integro-differential equations," Computers \& Mathematics with Applications, vol. 62, no. 5, pp. 2259-2272, 2011.

[8] V. Kavitha, P. Z. Wang, and R. Murugesu, "Existence of weighted pseudo almost automorphic mild solutions to fractional integro-differential equations," Journal of Fractional Calculus and Applications, vol. 4, pp. 37-55, 2013.

[9] C. Lizama and G. M. N’Guérékata, "Bounded mild solutions for semilinear integro differential equations in Banach spaces," Integral Equations and Operator Theory, vol. 68, no. 2, pp. 207227, 2010 .

[10] I. Mishra and D. Bahuguna, "Weighted pseudo almost automorphic solution of an integro-differential equation, with weighted Stepanov-like pseudo almost automorphic forcing term," Applied Mathematics and Computation, vol. 219, no. 10, pp. 5345-5355, 2013.

[11] Z. Šmarda, "Bounds of solutions of integrodifferential equations," Abstract and Applied Analysis, vol. 2011, Article ID 571795, 7 pages, 2011.

[12] Y. L. Zhao, L. Huang, X. B. Wang, and X. Y. Zhu, "Existence of solutions for fractional integrodifferential equation with multipoint boundary value problem in banach spaces," Abstract and Applied Analysis, vol. 2012, Article ID 172963, 19 pages, 2012.

[13] A. Lunardi, Analytic Semigroups and Optimal Regularity in Parabolic Problems, vol. 16 of Progress in Nonlinear Differential Equations and Their Applications, Birkhäuser, Basel, Switzerland, 1995.

[14] E. Bazhlekova, Fractional evolution equation in Banach spaces [Ph.D. thesis], Eindhoven University of Techology, 2001.

[15] E. Cuesta, "Asymptotic behaviour of the solutions of fractional integro-differential equations and some time discretizations," Discrete and Continuous Dynamical Systems A, supplement, pp. 277-285, 2007.

[16] I. Podlubny, Fractional Differential Equations, vol. 198 of Mathematics in Science and Engineering, Academic Press, New York, NY, USA, 1999.

[17] W. A. Coppel, Dichotomies in Stability Theory, Springer, Berlin, Germany, 1978.
[18] T. Diagana and G. M. N’Guérékata, "Pseudo almost periodic mild solutions to hyperbolic evolution equations in intermediate Banach spaces," Applicable Analysis, vol. 85, no. 6-7, pp. 769780, 2006.

[19] H.-S. Ding, J. Liang, G. M. N’Guérékata, and T.-J. Xiao, “Mild pseudo-almost periodic solutions of nonautonomous semilinear evolution equations," Mathematical and Computer Modelling, vol. 45, no. 5-6, pp. 579-584, 2007.

[20] K.-J. Engel and R. Nagel, One-Parameter Semigroups for Linear Evolution Equations, Springer, New York, NY, USA, 1999.

[21] Z. R. Hu and Z. Jin, "Stepanov-like pseudo almost automorphic mild solutions to nonautonomous evolution equations," Nonlinear Analysis. Theory, Methods \& Applications A, vol. 71, no. 5-6, pp. 2349-2360, 2009.

[22] H. L. Chen, "Antiperiodic wavelets," Journal of Computational Mathematics on Numerical Methods, Analysis and Applications, vol. 14, no. 1, pp. 32-39, 1996.

[23] G. M. N’Guérékata and V. Valmorin, "Antiperiodic solutions of semilinear integrodifferential equations in Banach spaces," Applied Mathematics and Computation, vol. 218, no. 22, pp. 11118-11124, 2012.

[24] F. Y. Wei and K. Wang, "An asymptotically periodic logistic equation," Journal of Biomathematics, vol. 20, no. 4, pp. 399-405, 2005.

[25] T. Diagana, "Doubly-weighted pseudo almost periodic functions," African Diaspora Journal of Mathematics, vol. 12, no. 1, pp. 121-136, 2011.

[26] H.-S. Ding, W. Long, and G. M. N’Guérékata, "Existence of pseudo almost periodic solutions for a class of partial functional differential equations," Electronic Journal of Differential Equations, no. 104, pp. 1-14, 2013.

[27] L.-L. Zhang and H.-X. Li, "Weighted pseudo-almost periodic solutions for some abstract differential equations with uniform continuity," Bulletin of the Australian Mathematical Society, vol. 82, no. 3, pp. 424-436, 2010.

[28] T. Diagana, "Existence of doubly-weighted pseudo almost periodic solutions to non-autonomous differential equations," Electronic Journal of Differential Equations, no. 28, pp. 1-15, 2011.

[29] A. A. Pankov, Bounded and Almost Periodic Solutions of Nonlinear Operator Differential Equations, Kluwer Academic Publishers, Dordrecht, The Netherlands, 1990.

[30] C. Cuevas and C. Lizama, "Almost automorphic solutions to a class of semilinear fractional differential equations," Applied Mathematics Letters, vol. 21, no. 12, pp. 1315-1319, 2008.

[31] P. Acquistapace and B. Terreni, "A unified approach to abstract linear nonautonomous parabolic equations," Rendiconti del Seminario Matematico della Università di Padova, vol. 78, pp. 47-107, 1987.

[32] P. Acquistapace, "Evolution operators and strong solutions of abstract linear parabolic equations," Differential and Integral Equations, vol. 1, no. 4, pp. 433-457, 1988.

[33] T. Diagana, "Stepanov-like pseudo-almost periodicity and its applications to some nonautonomous differential equations," Nonlinear Analysis. Theory, Methods \& Applications A, vol. 69, no. 12, pp. 4277-4285, 2008. 


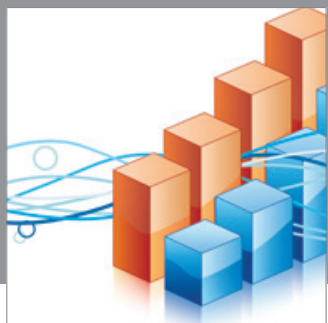

Advances in

Operations Research

mansans

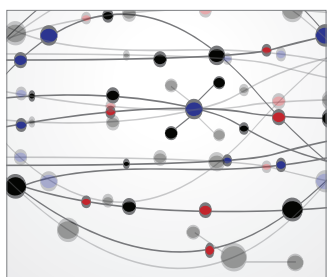

The Scientific World Journal
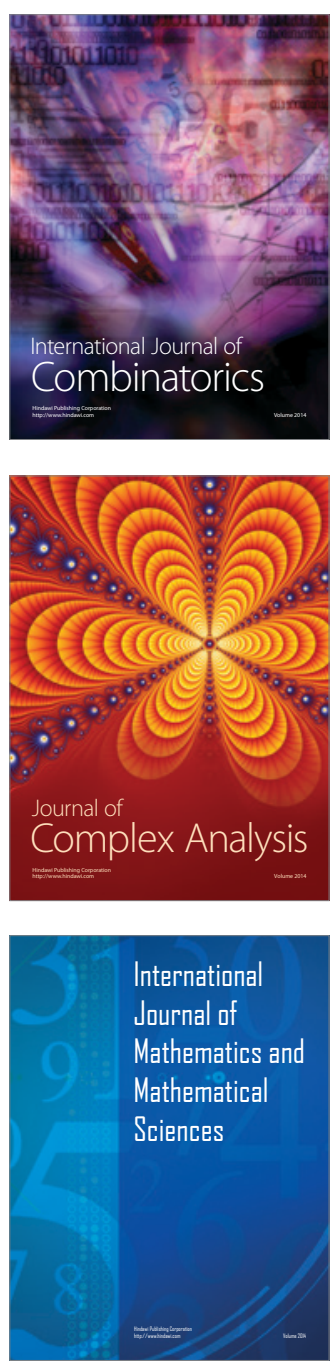
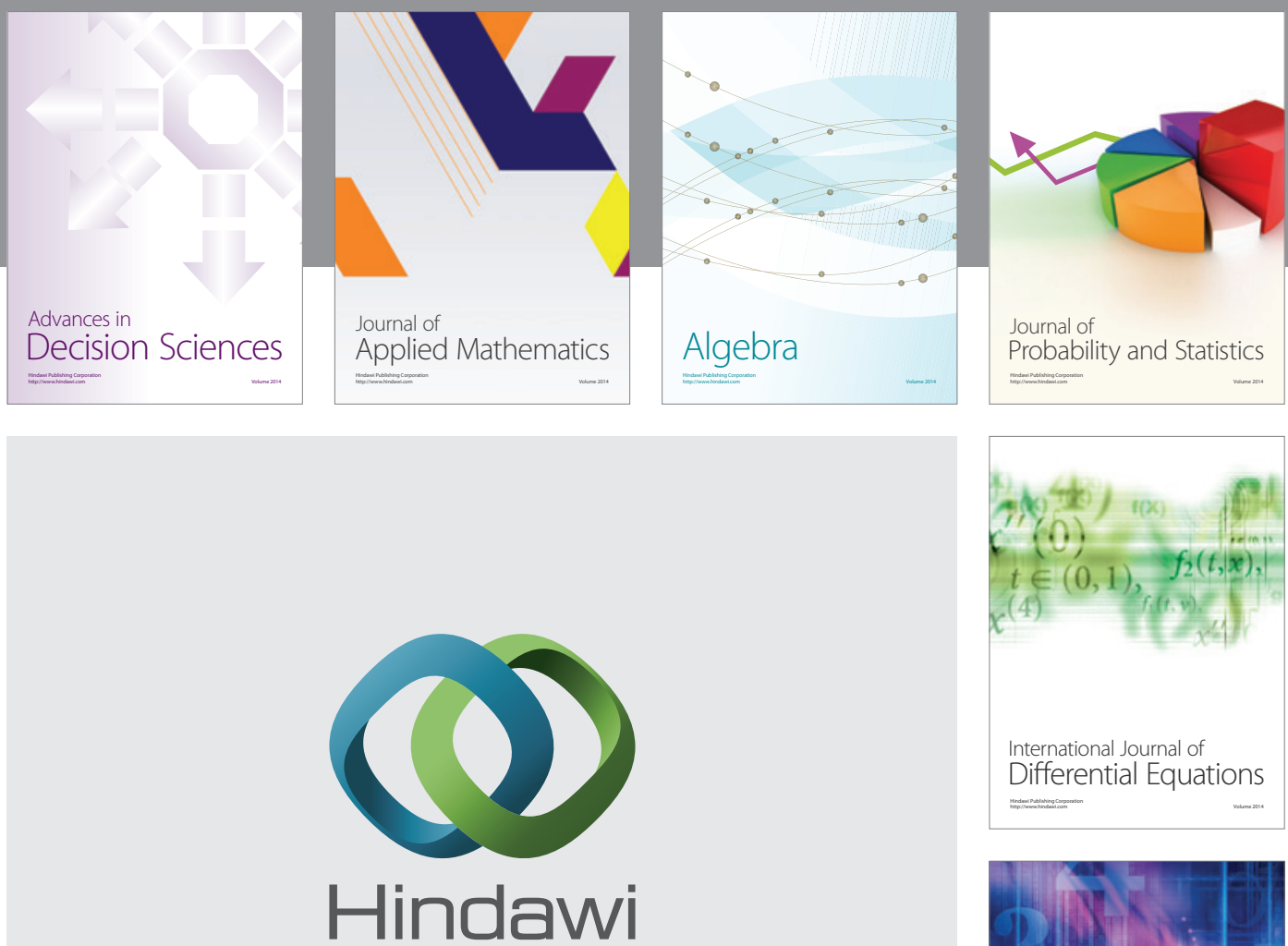

Submit your manuscripts at http://www.hindawi.com
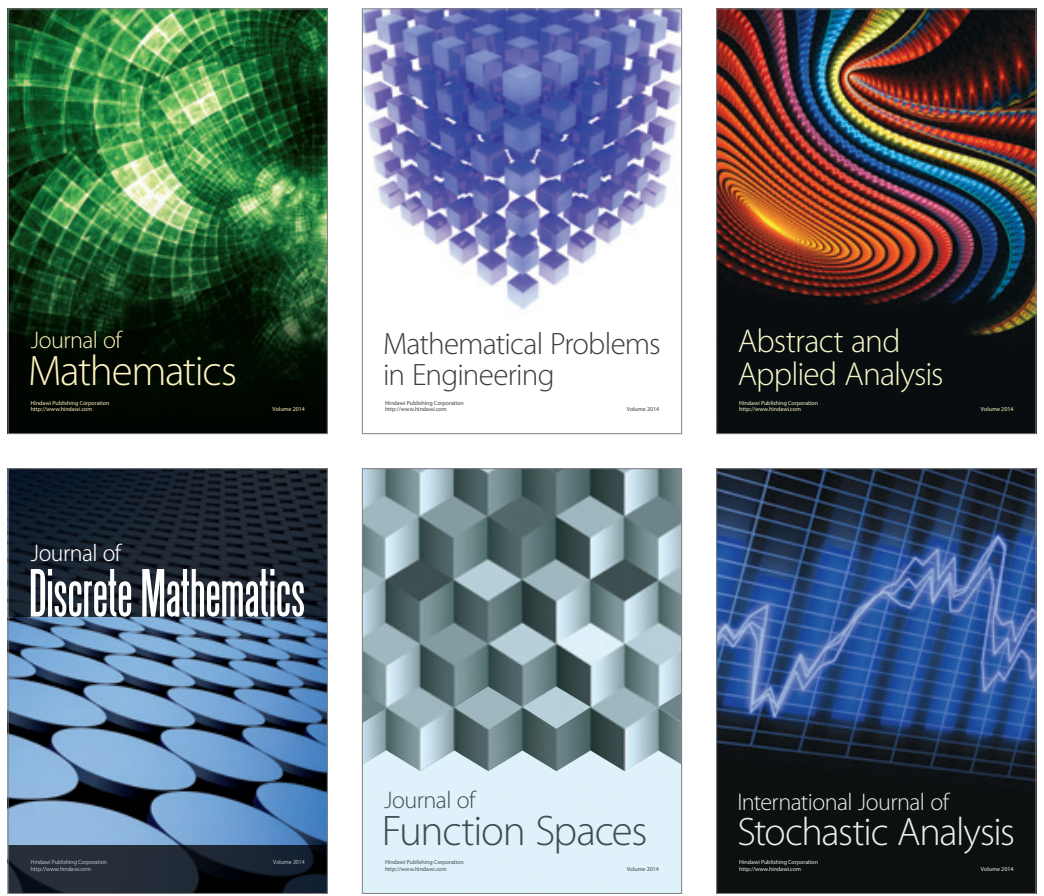

Journal of

Function Spaces

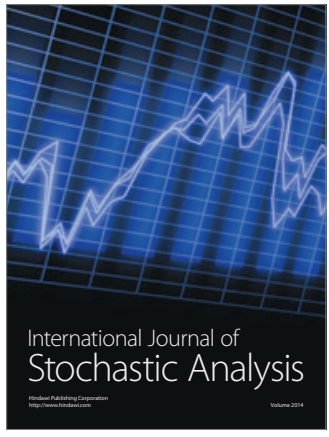

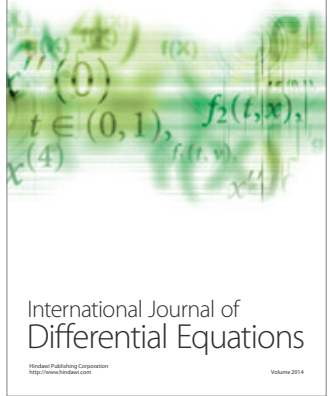
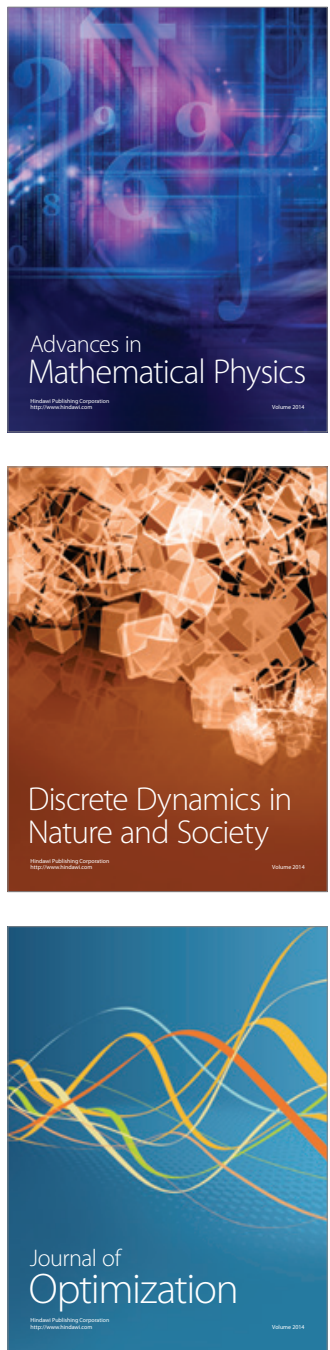